\title{
Le nouvel article 299 paragraphe 2 du traité instituant la Communauté européenne
}

Vers un approfondissement de la différenciation juridique en faveur des régions ultrapériphériques?

Danielle Perrot

\section{(2) OpenEdition}

Journals

Édition électronique

URL : http://journals.openedition.org/plc/350

DOI : $10.4000 /$ plc.350

ISSN : 2117-5209

Éditeur

L'Harmattan

\section{Édition imprimée}

Date de publication : 1 janvier 2000

Pagination : 111-152

ISSN : 1279-8657

\section{Référence électronique}

Danielle Perrot, « Le nouvel article 299 paragraphe 2 du traité instituant la Communauté européenne », Pouvoirs dans la Caraibe [En ligne], 12 | 2000, mis en ligne le 04 mars 2011, consulté le 30 avril 2019. URL : http://journals.openedition.org/plc/350; DOI : 10.4000/plc.350 


\title{
Le nouvel article 299 paragraphe 2 du traité instituant la Communauté européenne Vers un approfondissement de la différenciation juridique en faveur des régions ultra-périphériques ?
}

\author{
par Danielle PERROT ${ }^{*}$ \\ Chaire Jean Monnet \\ Maître de conférences (Droit Public) \\ Université des Antilles et de la Guyane
}

Le point 55 de l'article 2 du traité d'Amsterdam opère une réécriture de l'article 227 paragraphe 2 du traité initial, devenu l'article 299 paragraphe 2 dans la «version consolidée » du traité de Rome, entrée en vigueur le $1^{\text {er }}$ mai $1999^{1}$. La réforme du droit primaire à ce sujet, réclamée par les responsables des Exécutifs de sept Régions non européennes de la Communauté (Açores, Canaries, Guadeloupe, Guyane, Madère, Martinique, Réunion), opère d'emblée une unification de leur statut. Mais par-delà la compilation d'instruments auparavant dispersés, frappe surtout la volonté politique des auteurs de la nouvelle rédaction d'affirmer la légitimité de la différenciation juridique concernant ces régions : l'alinéa 2 en énonce abondamment les motifs et l'alinéa 3 fournit une liste indicative de ses domaines d'intervention. Cela rencontre les aspirations de ceux qui ont cherché à faire repousser les limites à la différenciation découlant de la rédaction de l'ex-article 227 paragraphe 2 concernant les DOM, tout en voulant

* La présente étude se situe dans le prolongement d'une réflexion amorcée sur ce sujet depuis 1995 au sein d'un groupe de recherche du Département Europe/DOM du CRPLC. Concernant la participation des juristes, on note particulièrement parmi les enseignants-chercheurs magistraux: E. JOS ; et notamment parmi les doctorants : M.-C. Dintimille ; B. Gaspard ; F. Miatti (†) ; J.-M. Ragald.

1. Union européenne, Traité d'Amsterdam, Luxembourg, OPOCE, 1997, p. 49 ; Union européenne, Versions consolidées du traité sur l'Union européenne et du Traité instituant la Communauté européenne, article 299 CE (ex-article 227 CE), Luxembourg, OPOCE, 1997, p. 159. 
préserver les avantages de l'intégration ${ }^{2}$. Mais la nouvelle stipulation suscite des incertitudes sur le degré de différenciation désormais licite, notamment parce que la fin de l'alinéa 4 de l'article 299 paragraphe 2 rappelle les autorités communautaires à la préservation de «l'intégrité et (de) la cohérence de l'ordre juridique communautaire $»^{3}$.

Répondre à cette interrogation nécessite de souligner que le statut communautaire de ces régions demeure placé sous le signe du principe de l'intégration. En effet, le nouvel article 299 paragraphe 2 reprend à son alinéa 1er, ce qui découle déjà du paragraphe 1er du même article : celui-ci énumère les États auxquels s'applique le traité ; celui-là rappelle que le traité s'applique à sept régions comprises dans des États membres. Par-delà l'effet de redondance, cette insistance liminaire trouve son écho en conclusion du paragraphe 2, avant que ses paragraphes ultérieurs ne recensent des statuts plus hétérodoxes: l'application partielle du droit communautaire, l'exclusion de son champ d'application ou l'association à la Communauté.

Surtout, l'alinéa 2 du paragraphe 2 amorce l'énoncé de la compétence du Conseil à arrêter des «mesures spécifiques » en l'articulant avec le rappel du principe d'intégration à l'alinéa précédent, par l'adverbe «Toutefois, ...». Cette restriction étant d'interprétation étroite, la mise en œuvre du traité peut valablement

2. On trouvera les réflexions du groupe de recherche du CRPLC à ce propos dans cette Revue $(P D L C)$ n ${ }^{\circ}$ 8-9, 1996-97, rubrique «Forum », p. 203-230. Voir aussi par exemple la reproduction de formulations proposées par les responsables des Exécutifs des sept Régions concernées, in Loïc Grard et Arnaud De Raulin (dir.), Le développement des DOM et la Communauté européenne, La Documentation française, coll. «Les études », 1998, annexe, p. 173 et s.

3. Sur les interrogations suscitées sur ce point par la nouvelle rédaction, voir notamment, Jacques Ziller, "Champ d'application du droit communautaire Application territoriale », Fascicule 470, Juris-classeur Europe, 3, 1998, n 79, p. 24 ; Loïc Grard, "Les départements d'outre-mer après les traités de Maastricht et d'Amsterdam », in Loïc Grard et Arnaud De Raulin (dir.), op. cit., particulièrement p. 52 ; Fabien Brial, « La place des régions ultrapériphériques au sein de l'Union européenne », Cah. Dr. Eur., n ${ }^{\circ}$ 5-6, 1998, particulièrement p. 655 et s ; Ismaël Omarjee, "Le traité d'Amsterdam et l'avenir de la politique de différenciation en faveur des départements français d'outre-mer », Rev. trim. de dr. eur., oct.-déc. 1998, particulièrement, p. 526 et $\mathrm{s}$. 
consister en dispositifs de droit commun, ce que confirme la mention selon laquelle les «mesures spécifiques » visent, «en particulier, à fixer les conditions de l'application du... traité... ${ }^{4}$ : ainsi l'édiction d'actes s'écartant du droit commun ne constitue pas la seule modalité possible. Dans les cas où le Conseil fait le choix d'édicter des règles d'application générale, sans particularisation spatiale, et qu'il opte en faveur du droit commun considéré comme adéquat pour les régions ultramarines, comme pour tous autres espaces communautaires, l'absence d'application différenciée serait seulement passible d'un contrôle contentieux restreint, celui de l'erreur manifeste d'appréciation ${ }^{5}$; la motivation doit certes porter sur l'opportunité, voire la nécessité d'arrêter des dispositions prises à l'échelle de la Communauté (voir article $253 \mathrm{CE}$; ex-article 190), mais l'alinéa $1^{\mathrm{er}} \mathrm{du}$ nouvel article 299 paragraphe 2 - de même que son paragraphe $1^{\text {er }}-$ suffit à en fonder l'application outre-mer sans justification supplémentaire. De tels actes se situeraient dans le prolongement des aspirations exprimées par les responsables des Exécutifs des sept Régions concernées qui ont tenu à ne pas remettre en cause le principe d'intégration.

4. Il ne semble pas possible de tirer des conclusions du changement de rédaction par rapport à celle de l'ex-article $227 \S 2$ al. 2 (« conditions $d$ 'application... du ... traité », selon la rédaction initiale en langue française) : la confrontation de diverses versions linguistiques du traité d'Amsterdam permet de constater que l'une reproduit la formule précédente (versions successives en langue portugaise : «condiçoes de aplicação »), telle autre opère une variation proche de celle constatée en français (versions successives en langue espagnole : «conditiones de aplicación », puis « conditiones para la aplicación »), et une troisième semble effectuer un changement inverse (versions successives en langue anglaise : "conditions under which the other provisions of this Treaty are to apply », puis « conditions of application »). Les «textes établis dans chacune de ces langues faisant également foi» (Traité d'Amsterdam, art. 15, in Union européenne, Traité d'Amsterdam, Luxembourg, OPOCE, 1997, p. 79), il n'y a pas lieu de considérer que l'un exprime mieux que les autres la volonté des Parties.

N.B. : Parmi beaucoup d'autres, la confrontation des versions linguistiques constitue l'une des contributions de Franck Miatti aux travaux du groupe de recherche du CRPLC sur la révision du statut communautaire des régions ultrapériphériques.

5. CJCE, 26 mars 1987, Coopérative Agricole d'Approvisionnement des Avirons, aff. 58/86, point 17, Rec. p. 1525. 
Pourtant, l'insistance des auteurs du texte sur les raisons d'arrêter des dispositifs différenciés, eu égard aux «caractéristiques et contraintes particulières » de ces régions (alinéa 4), témoigne du souci de permettre des dispositifs juridiques dont le contenu différerait de ceux qui sont applicables dans l'ensemble de la Communauté.

Certes, les contours précis des expressions «intégrité » et «cohérence de l'ordre juridique communautaire» ne sont pas forcément aisés à cerner en tant que limites qu'il serait illicite de transgresser. La formulation retenue exige au moins de considérer la disposition à interpréter comme participant d'un système juridique organisé, auquel la Cour de justice se réfère pour interpréter les normes qui y sont rattachées ${ }^{6}$. Un premier mode de conciliation peut alors être trouvé dans le cadre des possibilités offertes par le droit communautaire commun: l'intégration juridique n'impliquant pas l'uniformité du contenu des normes, les régions ultrapériphériques bénéficient au moins des mêmes possibilités de différenciation que celles n'ayant pas fait l'objet de mention particulière dans les différents paragraphes de l'article 299 (I). Se pose alors la question d'une différenciation propre à la catégorie de régions ultrapériphériques : si elle est licite, on pourra parler d'un statut particulier autorisant un degré d'altération du principe d'intégration plus élevé qu'à l'égard de l'ensemble des régions européennes de la Communauté (II).

\section{I. - LA DIFFERENCIATION COMPATIBLE AVEC LE DROIT COMMUN DE L'INTEGRATION}

La conciliation de l'impératif d'adéquation à la situation de ces régions avec le principe d'intégration peut découler des larges possibilités de différenciation offertes par le droit communautaire commun (A) ; la contrepartie en est alors que l'on ne saurait, pour les

6. L'interprétation d'une disposition communautaire nécessite «d'envisager à la fois l'esprit, l'économie et les termes (de celle-ci) compte tenu du système du traité et des finalités qui lui sont propres » (CJCE, 21 févr. 1973, Europemballage Corporation et Continental Can, aff. 6/72, point 22, Rec. p. 215). 
adopter, échapper à des exigences procédurales souvent complexes (B).

\section{A. - Les larges possibilités de différenciation permises par le droit commun}

Outre les cas de dérogations expressément prévues en droit primaire et dont l'exploitation n'est pas circonscrite à une zone prédéterminée, le droit communautaire commun a souvent permis une mise en œuvre diversifiée du traité par le droit dérivé. Cette approche dite «modulatoire », utilisable envers bien d'autres régions de la Communauté, peut se déployer pleinement pour l'application des alinéas 2 et 3 du nouvel article 299 § 2, qui insistent sur la nécessité de la différenciation, sans porter aucunement atteinte au respect dû à l'intégrité de l'ordre juridique communautaire?

\section{1. - La démarche de modulation}

L'alinéa 2 du nouvel article $299 \S 2$ énonce les traits des sept régions concernées, dont il doit être tenu compte lorsqu'il y a lieu d'arrêter à leur intention des mesures dites «spécifiques». Cette énumération des motifs de la différenciation juridique conduit à des difficultés de compréhension qui se dégageaient déjà de la lecture de la Déclaration $\mathrm{n}^{\circ} 26$ «relative aux régions ultrapériphériques de la Communauté », jointe au traité CE par le traité de Maastricht ${ }^{8}$.

7. Pour une présentation de «l'approche modulatoire», voir par ex. : CharlesMichel Geurts, «Les DOM et l'approche globale communautaire des régions ultrapériphériques », in E. Jos et D. Perrot (dir.): L'Outre-Mer et l'Europe communautaire - Quelle insertion? Pour quel développement?, Economica, coll. Collectivités territoriales, 1994, particulièrement p. 378 et $\mathrm{s}$.

8. Cependant la rédaction de ce texte, indique que « la Conférence reconnaît» l'existence d'une situation caractérisée par de nombreux facteurs portant «lourdement préjudice à leur développement économique et social » (al. $\left.1^{\text {er }}\right)$; elle n'induit pas aussi nettement que l'alinéa 2 du nouvel article $299 \S 2$, un énoncé de la motivation des 
L'auteur du droit dérivé doit tenir compte de la «situation économique et sociale structurelle » des régions concernées « qui est aggravée » par une série de facteurs défavorables que l'on peut être tenté de qualifier aussi de structurels : les premiers se présentent comme caractérisant ces régions de façon irréductible à celles de l'Europe communautaire et semblent inaccessibles à toute démarche curative - éloignement, insularité, faible superficie, relief et climat difficiles - ; le dernier «leur dépendance économique vis-à-vis d'un petit nombre de produits »- tout en apparaissant durable, n'exclut pas une recherche de remèdes. Au surplus, l'énoncé de ces facteurs aggravants insiste sur leur « permanence et (leur) combinaison (qui) nuisent gravement au développement de ces régions », ce qui évoque le premier membre de phrase : « la situation économique et sociale structurelle ». On peut donc supposer que les actes porteurs de normes diversifiées à l'intention de ces régions pourront pour partie tirer leur motivation de l'existence de difficultés rencontrées, quoiqu'avec des degrés différents, dans des régions européennes de la Communauté, alors que d'autres se baseront sur des caractéristiques propres aux régions ultramarines, considérées comme facteurs aggravants.

L'expérience de la mise en œuvre de l'ex-article 227 § 2 permet de souligner que, dans la première catégorie, peuvent prendre place des actes aménageant une différenciation concernant l'ensemble des zones communautaires désavantagées. Ce procédé est bien connu en ce qui concerne, par exemple, le droit dérivé relatif à l'agriculture de montagne et de certaines zones défavorisées ${ }^{9}$, les communications de

actes subséquents de droit dérivé. Son caractère insatisfaisant a été mis en évidence ; voir Emmanuel Jos: "La Déclaration du Traité de Maastricht sur les régions ultrapériphériques de la Communauté : essai d'appréciation », in Richard Burton et Fred Réno (dir.), Les Antilles-Guyane au rendez-vous de l'Europe: Le grand tournant ?, Economica, coll. Caraïbe-Amérique latine, 1994, spécialement p. 264 et s.

9. V. Directive du Conseil $\mathrm{n}^{\circ} 75 / 268$ du 28 avril 1975 sur l'agriculture de montagne et certaines zones défavorisées (JOCE L 128, 19 mai 1975, p. 1 et s.). Ce dispositif avait fait l'objet d'une refonte au titre IX (art. 17 et suivants) du règl. n $^{\circ}$ 950/97 du Conseil du 20 mai 1997 (JOCE L 142, 2 juin 1997, p. 1) qui se trouve à présent abrogé et remplacé par les dispositions pertinentes du règl. n ${ }^{\circ}$ 1257/1999 du Conseil, 17 mai 1999, concernant le soutien au développement rural par le FEOGA..., articles 17 et suivants (JOCE L 160, 26 juin 1999, p. 80). 
la Commission en matière d'aides d'État à finalité régionale ${ }^{10}$, l'inscription des régions ultramarines parmi celles qui bénéficient du degré supérieur d'intensité d'aides structurelles communautaires ${ }^{11}$. Lorsqu'il s'agit de décalquer pour l'outre-mer un dispositif initialement imaginé à destination de zones particularisées d'Europe ${ }^{12}$, c'est l'extension du bénéfice d'une norme déjà précédemment élaborée qui trouve sa justification dans les caractéristiques des régions nouvellement concernées, non le principe du caractère sélectif de son application.

Semble alors demeurer une place pour la prise en compte de facteurs propres aux régions ultramarines, en tant que handicaps permanents et cumulés, justifiant un contenu véritablement spécifique, au sens fort du terme, à finalité compensatrice de ces désavantages irréductibles. Au demeurant, les handicaps des diverses régions concernées peuvent varier : la mention à l'alinéa 4 du nouvel article $299 \S 2$ de la nécessité de tenir compte des «caractéristiques et contraintes particulières des régions ultrapériphériques... » autorise à considérer tantôt celles qui les particularisent collectivement par

10. V. Communication de la Commission sur la méthode pour l'application de l'article 92 paragraphe 3 sous a) et c) aux aides régionales (88/C 212/02), JOCE C 212, 12 août 1988, p. 2 ; Lignes directrices concernant les aides d'État à finalité régionale, JOCE C 74, 10 mars 1998, p. 6.

11. V. Régl. $n^{\circ} 2081 / 93$ du Conseil du 20 juillet 1993 modifiant le régl. n 2052/88 concernant les missions des Fonds à finalité structurelle..., Annexe I : Régions concernées par l'objectif n 1, JOCE L 193, 31 juillet 1993, p. 5 ; Règl. n 1260/1999 du Conseil, 21 juin 1999, portant dispositions générales sur les Fonds structurels, art. 3 $\S 1$ al. 2, JOCE L 161, 26 juin 1999, p. 1.

12. V. Directive (77/388) du Conseil du 17 mai 1977 concernant le système commun de taxe à la valeur ajoutée et son article 3 paragraphe 2 initial (JOCE L 145, 13 juin 1977, p. 1), puis Onzième directive (80/368) du Conseil du 26 mars 1980 sur l'exclusion des DOM du champ d'application de la directive 77/388 (JOCE L 90, 3 avril 1980, p. 41).

V. Directive (90/675) du Conseil du 10 décembre 1990 sur l'organisation des contrôles vétérinaires pour les produits en provenance des pays tiers (art. 17), JOCE L 373, 31 décembre 1990, p. 1 ; et la confirmation in Directive (91/496) du Conseil du 15 juillet 1991 (art. 13), JOCE L 268, 24 septembre 1991, p. 56 ; puis la reprise in Régl. n ${ }^{\circ} 3763 / 91$ du Conseil du 16 décembre 1991 «portant mesures spécifiques concernant certains produits agricoles en faveur des départements français d'outremer », JOCE L 356, 24 décembre 1991, p. 1. 
rapport aux régions européennes, tantôt celles qui ne sont propres qu'à certaines d'entre elles; d'ailleurs, en énumérant à l'alinéa 2 les désavantages qui les frappent, les auteurs du texte ne pouvaient ignorer que tous ces facteurs aggravants ne se rencontrent pas dans chacune des régions concernées : elles ne sont pas toutes sujettes aux risques cycloniques, la Guyane n'est caractérisée ni par la faible superficie, ni par l'insularité... Et leur prise en considération de façon combinée avec des indicateurs économiques et sociaux plus classiques doit conduire à une sélectivité dans l'application des dispositifs arrêtés ${ }^{13}$. Il est pourtant arrivé qu'un mécanisme initialement institué en considération de la situation de régions ultramarines soit transposé vers telles régions européennes connaissant certes des difficultés, mais échappant aux caractéristiques irréductibles de l' «ultrapériphérie $»^{14}$. Cela rappelle que, malgré l'emploi fréquent dans le vocabulaire juridique communautaire de l'expression «mesure spécifique » à propos de l'outre-mer, elle ne doit pas être toujours comprise au sens de dispositif exclusivement réservé aux régions ultrapériphériques. Bien souvent, on se situe dans le cadre des possibilités de différenciation permises par le droit commun, d'une démarche de modulation qui vaut pour l'ensemble communautaire.

13. L'existence d'une activité exportatrice significative dans le secteur de la pêche de certaines de ces régions a conduit à rechercher l'atténuation de l'impact de handicaps irréductibles, par un « régime de compensation des surcoûts induits par l'ultrapériphéricité pour l'écoulement de certains produits de la pêche des Açores, de Madère et du département français de la Guyane » (la motivation fait état du « coût des transports des produits de la pêche vers les marchés, du fait de l'éloignement et de l'isolement des régions ultrapériphériques » : Régl. $\mathrm{n}^{\circ}$ 1503/94 du Conseil du 27 juin 1994, JOCE L 162, 30 juin 1994, p. 8 ; régl. n 2337/95 du Conseil du 2 octobre 1995, JOCE L 236, 5 octobre 1995, p. 2. Pour la période 1998-2001, le règlement $\mathrm{n}^{\circ} 1587 / 98$ du Conseil du 17 juillet 1998 concerne également la Réunion (JOCE L 208, 24 juill. 1998, p. 1).

14. V. sur l'approvisionnement en produits agricoles, régl. $\mathrm{n}^{\circ}$ 3763/91 du Conseil du 16 décembre 1991 «portant mesures spécifiques concernant certains produits agricoles en faveur des départements français d'outre-mer », art. 2 et s., JOCE L 356, 24 décembre 1991, p. 1 ; puis, régl. n 2019/93 du Conseil du 19 mai 1993 «portant mesures spécifiques concernant certains produits agricoles en faveur des îles mineures de la mer Égée », art. 2 et s., JOCE L 184, 27 juill. 1993, p. 1. 
Cela se confirme à la lecture de l'alinéa 3 de l'article 299 § 2 qui comporte une énumération non limitative des domaines d'intervention des mesures dites spécifiques. Il s'agit des «domaines tels que les politiques douanières et commerciales, la politique fiscale, les zones franches, les politiques dans les domaines de l'agriculture et de la pêche, les conditions d'approvisionnement en matières premières et en biens de consommation de première nécessité, les aides d'État et les conditions d'accès aux fonds structurels et aux programmes horizontaux de la Communauté ». La confrontation de cette liste avec les normes de droit primaire concernées montre qu'il peut être souvent fait appel aux potentialités diverses de mise en œuvre du droit commun, avant de songer à des modalités supérieures de différenciation.

C'était particulièrement net en ce qui concerne des matières énoncées à l'alinéa 1er de l'ex-article 227 § 2, pour lesquelles il n'était pas question d'écarter le droit primaire commun. Les auteurs du droit dérivé disposent d'un large pouvoir d'appréciation pour mener la politique économique communautaire en matière d'agriculture (et de pêche), en matière de règles de concurrence : des dispositifs différenciés peuvent découler de la prise en considération de circonstances propres aux diverses régions communautaires. Cette démarche a également été privilégiée pour des domaines non expressément désignés à cet alinéa 1er primitif : concernant la fiscalité ou l'accès aux fonds structurels, les plus nombreux des actes arrêtés ne prévoient pas un traitement foncièrement original de ces régions ${ }^{15}$. En

15. En matière fiscale, outre le cas de la TVA précité, voir celui des accises [ex. : Directive (92/83) du Conseil du 19 octobre 1992 concernant l'harmonisation des structures des droits d'accises sur l'alcool et les boissons alcooliques, art. 23-1), JOCE L 316, 31 octobre 1992, p. 21 et s.].

Du point de vue des financements structurels, les dispositifs en vigueur jusqu'en 1999, comme ceux prévus pour la période 2000-2006, sont largement modulés : classement des régions selon certains «objectifs », taux de cofinancement. Il serait même possible de créer un instrument structurel exclusivement orienté vers les régions ultramarines, à l'instar de celui qui concerne des États européens candidats, en se fondant sur l'actuel article 308 CE (ex-art. 235) ; voir Règl. n 1267/1999 du Conseil, 21 juin 1999, établissant un instrument structurel de préadhésion (ISPA) [JOCE L 161, 26 juin 1999, p.73]. 
outre, des réglementations communautaires, voire nationales, relatives à des conditions particulières d'approvisionnement - notamment en considération de l'insularité - se situent dans l'optique du droit commun qui autorise une adéquation à des situations diverses ${ }^{16}$.

La liste des domaines figurant à l'alinéa 3 du nouvel article 299 $\S 2$ ne semble donc pas reposer sur une analyse juridique du degré de différenciation admissible - ou non - sans mise à l'écart de l'application du droit primaire matériel. Elle apparaît comme un programme indicatif d'intervention du droit dérivé, élaboré en fonction de l'importance accordée sur le moment aux questions soumises aux instances communautaires. Les stipulations de droit matériel persistant très largement en l'état après la dernière révision, peut se poursuivre amplement l'exploitation de la malléabilité du droit commun, en pleine cohérence avec la réaffirmation de la priorité du principe d'intégration juridique des régions ultramarines, notamment au premier alinéa du nouvel article 299 § 2 .

Sans doute, les actes à venir dans cette perspective peuvent puiser leur motivation dans l'énoncé de difficultés énumérées à son alinéa 2 et instituer des dispositifs empêchant la perturbation du marché intérieur ou de telle politique commune à l'échelle de la dimension européenne de la Communauté ${ }^{17}$, ce à quoi invitent les derniers mots de son alinéa 4. A ce moment, comme antérieurement, ils trouvent facilement leur fondement dans des bases juridiques de droit commun, tout comme ceux qui exploiteraient à destination de régions ultrapériphériques les possibilités de dérogation permises de façon plus globale par le droit primaire.

16. Sur l'approvisionnement des DOM en produits agricoles, voir régl. $n^{\circ}$ 3763/91 du Conseil du 16 décembre 1991 précité. Concernant un dispositif interne sur l'approvisionnement en produits pétroliers de la République d'Irlande, v. CJCE, 10 juill. 1984, Campus Oil, aff. 72/83, Rec. p. 2727 [cf. CJCE, 30 nov. 1995, Esso SA c/ Comunidad Autonoma de Canarias, aff. C-134/94, Rec. p. I-4223].

17. En matière d'interdiction de « réexpédition vers le reste de la Communauté » de denrées notamment importées d'États tiers dans des DOM, voir régl. n 3763/91 du 16 décembre 1991 précité, art. 8. 


\section{2. - Le cas des dérogations expressément prévues en droit primaire}

Dans différentes hypothèses, le traité a expressément prévu parmi les stipulations de portée générale l'éventualité du recours à des dérogations. On est alors en présence d'une habilitation donnée par l'auteur du droit primaire à arrêter des dispositions de droit dérivé non seulement dissemblables les unes par rapport aux autres, mais éventuellement contradictoires avec une norme posée dans le traité luimême.

Il peut s'agir de clauses dérogatoires, figurant dans le traité, offrant aux États des possibilités de restreindre la portée de leurs obligations en matière d'édification du marché intérieur, pour des motifs non économiques (moralité publique, santé publique, ordre public.... ${ }^{18}$, sous réserve d'un contrôle contentieux ultérieur : il en est ainsi du maintien de mesures d'effet équivalant à des restrictions quantitatives [art. $30 \mathrm{CE}$ (ex-art. 36)] en ce qui concerne la libre circulation des marchandises, de la restriction de l'accès de ressortissants communautaires à leur territoire [art. 39 CE (ex-art. 48) $\S 3$, art. $46 \mathrm{CE}$ (ex-art. 56)] ou seulement à certains emplois ou activités professionnelles [art. $39 \mathrm{CE}$ (ex-art. 48) § 4, art. $45 \mathrm{CE}$ (exart. 55) al. $1^{\text {er }}$ en matière de libre circulation des personnes. Compte tenu des motifs pouvant être légitimement avancés, les dispositions décidées en ce sens au niveau national ont vocation à couvrir l'intégralité du territoire étatique ${ }^{19}$. Une mesure d'interdiction prise par l'un des États membres dont dépendent les régions ultrapériphériques, pleinement incluses dans l'espace communautaire, et qui exclurait l'une d'entre elles de son champ d'application - ou un acte qui ne concernerait que l'une des régions ultrapériphériques et non l'ensemble $\mathrm{du}$ territoire étatique - risquerait de ne pas satisfaire aux conditions de

18. Voir D. Waelbroeck et J. Bouckaert, «Exception de l'ordre public », in A. Barav et C. Philip, Dictionnaire juridique des Communautés européennes, PUF, 1993, p. 733.

19. Sur le principe de la détermination du champ spatial des libertés de circulation et des restrictions susceptibles d'y être apportées, voir CJCE, 28 oct. 1975, Rutili, aff. 36/75, Rec. p. 1219 , points 46 et suivants. 
licéité imposées par le droit communautaire, et particulièrement à celle tenant au caractère nécessaire et proportionné des mesures restrictives.

Surtout, le traité peut habiliter expressément le législateur communautaire à prévoir des «dispositions dérogatoires », de façon ponctuelle. C'est le cas dans des domaines ne figurant pas à l'énumération indicative de l'alinéa 3 de l'article 299 §. Ainsi, en matière électorale, le droit de vote et d'éligibilité de citoyens de l'Union aux élections municipales ou européennes dans l'État membre où ils résident peut s'exercer dans des conditions qui différent de celles qui s'appliquent aux ressortissants de cet État ; mais ce dernier ne peut établir ou maintenir ces restrictions que si le Conseil a arrêté des mesures dérogatoires « lorsque des problèmes spécifiques à un État membre le justifient $»^{20}$. On peut pourtant souligner que l'usage de cette faculté donnée au Conseil n'a pas consisté à autoriser l'exclusion totale de citoyens de l'Union de ce droit, même dans certaines parties circonscrites d'un État, mais seulement à en subordonner la reconnaissance à des conditions supplémentaires (allongement de la durée de résidence.... $)^{21}$. Le traité prévoit encore expressément l'éventualité de «dérogations temporaires» en matière d'environnement, à l'article $175 \mathrm{CE}$ (ex-art. $130 \mathrm{~S}) \S 5 \mathrm{1}^{\mathrm{er}}$ tiret. Ici, le droit primaire prédétermine la motivation - des coûts jugés disproportionnés pour les pouvoirs publics d'un État membre - mais permet une solution alternative ou cumulative - un soutien financier du Fonds de cohésion dont l'Espagne et le Portugal peuvent bénéficier -, de manière à rendre le recours à la dérogation aussi circonscrit que possible.

Dans le cas d'un acte dérogatoire de droit dérivé pris sur autorisation du traité, on pourrait admettre que, pour les régions ultramarines, le contenu de la dérogation aille plus loin que ce qui est arrêté envers des zones européennes, du moment que la différenciation supérieure est justifiée par les circonstances particulières rappelées par

20. Art. $19 \S 1$ et $\S 2$ CE (ex-art. 8 B $\S 1$ et $\S 2$ inséré par le traité de Maastricht).

21. Voir ainsi à propos des élections municipales au Luxembourg et dans certaines communes de la Belgique, la directive (94/80) du Conseil du 19 déc. 1994, art. 12, JOCE L 368, 31 déc. 1994, p. 38. 
l'alinéa 2 de l'article 299 § 2 CE, et tant qu'elle reste proportionnée à l'objectif poursuivi. Pour un acte de ce type, il serait approprié d'en avancer la justification dans des motifs inspirés du contenu de l'alinéa 2 de l'article $299 \S 2$; ce dernier pourrait même figurer dans les visas, à la condition d'y mentionner surtout la base juridique ayant expressément autorisé le législateur communautaire à déroger aux règles générales du traité.

Dès lors, comme à propos du droit dérivé relevant de l'approche dite «modulatoire », le recours à des bases juridiques de droit commun situe les régions ultramarines dans la droite ligne du respect de l'intégrité et de la cohérence de l'ordre juridique communautaire, ainsi que l'impose l'alinéa 4 de l'article 299 § 2. S'ensuit l'obligation d'observer les procédures d'élaboration des actes qui y sont prescrites, dont on a pu observer la complexité croissante, au fil des révisions successives.

\section{B. - Des exigences procédurales souvent complexes}

La base juridique pertinente de droit commun dans un domaine donné exige une procédure fréquemment plus solennelle que celle décrite au nouvel article $299 \S 2$ alinéa 2 du traité. Le schéma procédural énoncé - proposition de la Commission, simple consultation du Parlement, majorité qualifiée au Conseil - apparaît comme une survivance de la période où le Conseil était le législateur communautaire exclusif. Certes, les cas où subsiste la possibilité pour le Conseil de statuer seul, à la majorité qualifiée, peuvent concerner des domaines importants de l'action communautaire; mais ils deviennent moins nombreux. Lorsque le législateur communautaire veut mettre en œuvre les possibilités de différenciation permises par les stipulations de portée globale du traité en arrêtant des actes de premier niveau $^{22}$, il convient d'éviter la contestation contentieuse - émanant en

22. Les actes constituant le droit dérivé de premier niveau «trouvent directement leur base légale dans le traité »; les actes de deuxième niveau sont destinés à assurer l'exécution des premiers : V. Guy Isaac, Droit communautaire général, Armand Colin, 
particulier du Parlement européen en vue du respect de ses prérogatives $^{23}$ - qui suivrait l'édiction d'un acte selon une procédure simplifiée, en violation des prescriptions de la base juridique pertinente.

\section{1. - L'obligation de respecter des procédures plus solennelles}

Puisque l'élaboration d'actes de droit dérivé porteurs de normes juridiques indifférenciées doit suivre les prescriptions procédurales inscrites dans les bases juridiques pertinentes, les institutions doivent observer la même rigueur pour l'adoption d'actes mettant en œuvre soit une habilitation expresse à déroger au traité, soit une démarche de modulation du droit commun. A partir de la nécessité de se reporter au cas par cas à l'article qui fixe la procédure à suivre, on note une tendance à la généralisation de la procédure dite de co-décision, pour laquelle le traité d'Amsterdam a renforcé le poids du Parlement européen (nouvel art. $251 \mathrm{CE}$ ). Mais bien souvent, d'autres organes communautaires interviennent, ou encore le Conseil doit statuer à l'unanimité.

Cela se remarque, en ce qui concerne les cas précédemment identifiés où le traité autorise l'auteur du droit dérivé à arrêter des dérogations, selon la même procédure que des actes de mise en œuvre de la logique d'intégration. En matière d'environnement, l'article 175 $\S 1 \mathrm{CE}$ rend obligatoires la co-décision du Conseil et du Parlement et les consultations du Comité économique et social et du Comité des

Dalloz, $\mathcal{T}$ éd. 1999, p. 134 ; Rostane Mehdi, «Actes des institutions », in A. Barav et C. Philip, Dictionnaire juridique des Communautés européennes, PUF, 1993, p. 49. Sur ce point, on peut regretter que le débat amorcé au cours de la Conférence intergouvernementale de 1991 sur la hiérarchie des actes communautaires n'ait pas progressé pendant l'élaboration du traité d'Amsterdam ; V. déclaration $\mathrm{n}^{\circ} 16$ ajoutée par le traité de Maastricht au traité CE: Union Européenne, Recueil des Traités, Tome I, vol. I, Luxembourg, OPOCE, 1995, p. 664.

23. CJCE, 22 mai 1990, Parlement c/ Conseil, aff. C-70/88, Rec. p. I-2041; art. 173 al. $3 \mathrm{CE}$ issu du traité de Maastricht (actuellement art. 230 al. 3). 
régions ${ }^{24}$; cette procédure s'applique même lorsqu'un acte comporte des dérogations en tant que « dispositions appropriées » (article 175 $\S 5 \mathrm{CE}$ ). En ce qui concerne le droit de vote, l'article $19 \mathrm{CE}$ exige toujours, comme son devancier (ex-article $8 \mathrm{~B}$ ), que les modalités de mise en œuvre du traité, même non dérogatoires, soient adoptées à l'unanimité ; il ne saurait être question que les «dispositions dérogatoires» expressément prévues soient arrêtées selon une procédure plus souple, en ce qui concerne les citoyens européens de l'Union, et rien ne justifie la seule majorité qualifiée, en ce qui concerne telle région ultrapériphérique dont l'intégration juridique a fait l'objet d'une réaffirmation de principe.

Dans le cadre de la mise en œuvre de la démarche de modulation, on constate des exigences comparables. Parfois le Conseil doit statuer à l'unanimité ${ }^{25}$, mais il est alors fréquemment tenu par l'obligation de consulter un autre organe, voire d'obtenir un avis conforme du Parlement ${ }^{26}$. Il peut encore arriver que le Conseil statue seul à la majorité qualifiée au terme d'un dialogue avec le Parlement européen, selon la procédure dite de coopération, introduite par l'Acte

24. La révision de l'ex-article $130 \mathrm{~S} \S 1$ par le traité de Maastricht avait seulement imposé le respect de la procédure de coopération et la consultation du Comité économique et social.

25. Par delà le domaine de la citoyenneté ou celui des institutions [par ex. le Conseil peut modifier le nombre de membres d'une autre institution : la Commission (actuel art. $213 \S 1$ al. 2 CE), la Cour de justice, sur demande de celle-ci (art. 221 et $222 \mathrm{CE}$ )], l'unanimité peut être de règle en matière économique : directives concernant les régimes d'accès aux professions, lorsqu'elles conduisent à modifier des principes législatifs dans un État membre (actuel art. 47 § $2 \mathrm{CE}$ ) ; actes relatifs à la fiscalité (actuel art. $93 \mathrm{CE}) \ldots$

26. A titre d'exemple de la simple consultation du Parlement et du Comité économique et social, v. art. 93 CE précité (fiscalité) ; concernant l'avis conforme du Parlement, outre le domaine électoral (procédure uniforme d'élection de ses membres : actuel art. $190 \S 4 \mathrm{al} .2 \mathrm{CE}$ ), il demeure cumulativement exigé avec le vote unanime du Conseil dans le domaine des fonds à finalité structurelle qui intéresse les régions désavantagées, en ce qui concerne l'élaboration des actes les plus généraux (définition des missions, des objectifs prioritaires, organisation, efficacité et coordination : actuel art. $161 \mathrm{CE}$ ) ; sont alors aussi imposées les consultations du Comité économique et social et du Comité des régions. 
unique dans le traité $\mathrm{CEE}^{27}$. Par l'effet de la révision opérée par le traité d'Amsterdam, elle se trouve généralement remplacée par la procédure dite de co-décision, où le Conseil statue en principe à la majorité qualifiée, mais doit tenir compte du poids accru du Parlement $^{28}$ et respecter au cas par cas des formalités consultatives ${ }^{29}$.

Finalement, la mention insérée dans les dernières semaines de la négociation du traité d'Amsterdam quant au devoir de veiller au respect de l'intégrité et à la cohérence de l'ordre juridique communautaire se traduit, sur le plan procédural, par la reprise implicite de l'idée formulée dans des documents préparatoires, selon laquelle les institutions agissent «dans le cadre des procédures prévues

27. Actuellement, art. 252 CE. Pour l'essentiel, la procédure de coopération - non modifiée - reste cantonnée au domaine de l'Union économique et monétaire.

28. Il était en effet possible au Conseil de statuer seul dans le cas où une opposition du Parlement ne représentait qu'une majorité de suffrages et non la majorité absolue de ses membres (après échec du comité de conciliation ; voir ex-art.189 B § 6 CE en vigueur du $1^{\text {er }}$ novembre 1993 au 30 avril 1999) ; cette possibilité est supprimée (art. $251 \S 6 \mathrm{CE})$.

29. Des illustrations de l'accroissement du champ d'application de la procédure de co-décision peuvent se rencontrer notamment dans des domaines intéressant des régions défavorisées. Certains des actes relatifs à des fonds à finalité structurelle requièrent à présent cette exigence: pour le Feder, cette procédure ne fait pas disparaître les consultations obligatoires du Comité économique et social et du Comité des régions (art. 162 al. $1^{\text {er }} \mathrm{CE}$ ) ; pour le Fonds social, sont désormais imposées la codécision et les consultations du Comité économique et social et du Comité des régions (art. 162 al. 2 CE renvoyant à l'art. $148 \mathrm{CE}$ ). Dans le domaine des réseaux transeuropéens où l'action de la Communauté doit tenir compte des « régions insulaires... et périphériques » (ex-art. 129 B CE devenu art. 154 \& 2 CE), non seulement les orientations (ex-art. 129 D CE), mais aussi « les autres mesures » (nouvel art. $156 \mathrm{CE}$ ) sont désormais adoptées selon la procédure de co-décision ; les consultations du Comité économique et social et du Comité des régions demeurent requises.

Dans le domaine du marché intérieur, la procédure de l'article $251 \mathrm{CE}$ est prescrite pour l'adoption de directives relatives à l'exercice d'activités professionnelles indépendantes (art. $47 \S 2 \mathrm{CE}$; dans certains cas le Conseil doit statuer à l'unanimité : v. supra note 25), de la plupart des actes permettant de développer la politique commune de transport (art. $80 \mathrm{CE}$ renvoyant à l'art. $71 \mathrm{CE}$ ), de nombreux actes de rapprochement des législations en vue du fonctionnement du marché intérieur (art. 95 $\mathrm{CE})$. 
par le présent traité ${ }^{30}$. La possibilité du Conseil de statuer seul à la majorité qualifiée est donc plus restreinte que ne le laisse penser une première lecture.

\section{2. - Le champ limité de la seule exigence de majorité qualifiée au sein du Conseil}

La procédure décrite à l'article $299 \S 2$ se retrouve dans une série de bases juridiques de droit commun, touchant à des domaines intéressant particulièrement les régions ultrapériphériques, ainsi que le confirme la rédaction de l'alinéa 3 de cet article, tels que la politique agricole commune et la politique commune des pêches (art. $37 \mathrm{CE}$; ex-art. 43) - y compris les instruments financiers à vocation au moins partiellement structurelle comme le FEOGA et l'IFOP - et même les aides publiques internes (art. $89 \mathrm{CE}$; ex-art. 94) ${ }^{31}$. En ce qui concerne la poursuite de la réalisation du marché intérieur, à la sauvegarde duquel les institutions doivent veiller, comme à celle des politiques communes, l'article $14 \mathrm{CE}$ (ex-art. 7 A) renvoie à une série de bases juridiques dont certaines ne requièrent que la majorité qualifiée du Conseil ; il en est ainsi pour la fixation des droits du tarif douanier commun (art. $26 \mathrm{CE}$ ) - ce qui peut correspondre à la mention des « politiques douanières et commerciales » à l'alinéa 3 de l'article 299

30. Voir le «Document officieux (Compilation de textes à l'examen) 》 du 14 mai 1997 [SN 2555/97 : Chapitre 8, p. 22 de la subdivision SN/540/97 (C 41)] et le document «Consolidated draft Treaty texts » du 30 mai 1997 [SN 600/97 (C 101), p. 66]. En revanche, des documents antérieurs sont évasifs sur les procédures à observer : voir «Document de Dublin » du 5 décembre 1996 (« L’Union européenne, Aujourd'hui et demain »), point J. de la Partie B : Autres questions, p. 65. [Le document «Addendum to the Dublin II - General outline for a draft revision of the Treaties »(CONF/2500/96 ADD. 1, Brussels, 20 March 1997) n'aborde pas cette question].

31. La majorité qualifiée est de règle lorsque le Conseil prend «tous règlements utiles en vue de l'application » du traité. Lorsque, en revanche, il s'agit de déroger au droit primaire, l'unanimité au Conseil est requise (art. $88 \S 2$ al. $3 \mathrm{CE}$ ). 
$\S 2^{32}$, pour l'extension du bénéfice de la libre prestation de service à des ressortissants d'États tiers (art. $49 \mathrm{CE}$ ) et pour l'extension de la politique des transports à la navigation aérienne et maritime (art. 80 $\mathrm{CE}$ ).

L'on peut retenir de ces éléments que la démarche de modulation a particulièrement trouvé un terrain d'élection en matière agricole ou de pêche, ce qui tient sans doute à ce que les stipulations du traité assignent aux institutions une pluralité d'objectifs énoncés à l'article $33 \mathrm{CE}$ (ex-article 39), que le législateur doit concilier, dans l'exercice de son pouvoir discrétionnaire. Quand, antérieurement à la révision, un même acte prévoyait des dispositifs tantôt applicables dans les régions européennes, tantôt concernant seulement telles zones ultramarines, il visait la base juridique de droit commun en matière agricole $^{33}$. Quand il a été jugé bon d'arrêter des actes «spécifiques » à des régions ultramarines, il n'a pas été nécessaire de se fonder sur une base juridique concernant spécialement ces régions ${ }^{34}$, même quand il

32. On trouve aussi l'exigence du vote du Conseil à la majorité qualifiée, sans consultation obligatoire du Parlement européen, en matière de politique commerciale commune [art. 133 § 4 CE (ex-art. $113 \S 4)$; art. $300 \S 1$ al. 2 CE (ex-art. $228 \S 1$ al. 2)].

33. Ex. : Règl. $\mathrm{n}^{\circ} 1360 / 78$ du Conseil du 19 juin 1978 concernant les groupements de producteurs et leurs unions, art. $3 \S 2$, JOCE L166, 23 juin 1978, p. 1; Directive (90/675) du Conseil du 10 décembre 1990 fixant les principes relatifs à l'organisation des contrôles vétérinaires pour les produits en provenance des pays tiers introduits dans la Communauté, notamment art. 17, JOCE L 373, 31 décembre 1990, p. 1; Règl. $\mathrm{n}^{\circ}$ 2328/91 du Conseil du 15 juillet 1991 concernant l'amélioration de l'efficacité des structures de l'agriculture, art. $18 \S 1$ al. 2, JOCE L 218, 6 août 1991, p. $1 \ldots$

34. Ainsi, la directive (81/527) du Conseil du 30 juin 1981 concernant le développement de l'agriculture dans les départements français d'outre-mer (JOCE L 197, 20 juillet 1981, p. 38) comme le règlement $n^{\circ}$ 1503/94 du Conseil du 27 juin 1994 relatif à un régime de compensation des surcoûts induits par l'ultrapériphéricité pour l'écoulement de certains produits de la pêche des Açores, de Madère et du département français de la Guyane (JOCE L 162, 30 juin 1994, p. 8) visent alors l'article 43 (devenu art. $37 \mathrm{CE}$ ) du traité.

En revanche, avant que la Cour de justice ne se prononce sur le principe d'application intégrale du droit communautaire aux DOM, exprimé par l'article 227 $\S 1^{\mathrm{er}}$ et $\S 2$ CEE (CJCE, 10 octobre 1978, Hansen, affaire 148/77, point 9, Rec. p. 1787 et s.) nombre d'actes du Conseil visaient simultanément les articles 43 et 227 du traité : règl. $\mathrm{n}^{\circ} 1009 / 67 \mathrm{du} 18$ décembre 1967 portant organisation commune des marchés 
s'est agi de contourner au profit de produits typiques de départements d'outre-mer la rigidité de la liste des productions susceptibles de bénéficier des financements du FEOGA : cette liste pour l'essentiel élaborée en considération de réalités agricoles européennes n'a jamais compris le rhum agricole ${ }^{35}$, mais il a été estimé possible de recourir à la notion de filière ${ }^{36}$ en se fondant sur l'ex-article 43 (devenu art. 37 $\mathrm{CE}$ ), selon un raisonnement exploitable au profit de tout autre produit européen. Cette pratique consistant à privilégier le principe de la pleine intégration juridique des régions ultrapériphériques devrait se poursuivre, la procédure désormais inscrite à l'article 299 § étant de surcroît identique à celle exigée par l'article 37 du traité : même si la motivation d'un acte de droit dérivé peut s'inspirer de celui-là, le deuxième a toute chance d'être visé avant les considérants.

dans le secteur du sucre, JOCE 308, 18 décembre 1967, p. 1; règl. n ${ }^{\circ}$ 1929/75 du 22 juillet 1975 instituant un régime d'aide à la production pour les conserves d'ananas, JOCE L 198, 29 juillet 1975, p. 13 ; règl. $\mathrm{n}^{\circ} 1795 / 76$ du 20 juillet 1976 relatif aux conditions d'application de l'article 40 paragraphe 4 du traité aux départements français d'outre-mer, JOCE L 201, 27 juillet 1976, p. 5; règl. n ${ }^{\circ}$ 594/78 du 20 mars 1978 modifiant le règl. $\mathrm{n}^{\circ}$ 1418/76 portant organisation commune du marché du riz en ce qui concerne le département français de la Réunion, JOCE L 82, 29 mars 1978, p. 10.

35. L'article 6 point 25 a) du traité d'Amsterdam supprime la phrase de l'article 38 $\S 3$ du traité de Rome relative à l'habilitation conférée temporairement au Conseil d'y inscrire d'autres produits ; il renumérote également en annexe I, l'annexe II primitive comportant la liste des produits concernés, sans en modifier le contenu (Union européenne, Traité d'Amsterdam, Luxembourg, OPOCE, 1997, p. 60).

36. Après une motivation explicite en ce sens, au profit du rhum agricole [Annexe (point 8.3) à la Décision (89/687) du Conseil du 22 décembre 1989 instituant un programme d'options spécifiques à l'éloignement et à l'insularité des départements français d'outre-mer (Poséidom), JOCE L 399, 31 décembre 1989, p. 39], c'est encore à cette notion que se réfère de façon moins manifeste le Conseil pour décider d'aider des producteurs d'huiles essentielles : «des mesures de soutien doivent être... prises dans la limite de quantités déterminées... en faveur de certaines productions traditionnelles, telles que la vanille, le géranium ou le vétiver » $\left(10^{\mathrm{e}}\right.$ considérant du règlement $\mathrm{n}^{\circ}$ 2598/95 du Conseil du 30 octobre 1995 modifiant le règlement $\mathrm{n}^{\circ} 3763 / 91$ portant mesures spécifiques concernant certains produits agricoles en faveur des départements français d'outre-mer, JOCE L 267, 9 novembre 1995, p. 1; voir les modifications relatives à l'art. $13 \S 3$ et $15 \S 5$ ). 
S'agissant de l'accès facilité à des financements structurels pour les régions ultramarines dans la ligne de l'alinéa 3 de l'article 299 § 2, l'on retrouve la problématique de la mise en œuvre du droit primaire commun. Certes, pourrait se faire jour la tentation d'invoquer un précédent relatif à la reconstitution du potentiel de production après le passage de cyclones ${ }^{37}$ pour faire financer par le FEOGA - qui voit sa section Garantie de plus en plus sollicitée pour des financements naguère jugés structurel ${ }^{38}$ - des actions relevant plutôt du Feder. La raison pourrait en être que la procédure imposée est alors plus souple : pour le premier, l'article $162 \mathrm{CE}$ (ex-art. $130 \mathrm{E}$ ) alinéa 2 renvoie à l'article $37 \mathrm{CE}$ (ex-art. 43) qui n'exige que la consultation du Parlement, alors que, concernant le Feder, l'article 162 alinéa $1^{\text {er }} \mathrm{CE}$ réclame la co-décision (et les consultations du Comité économique et social et du Comité des régions); dans ce cas, comme pour toutes autres zones de la Communauté, le Parlement européen contesterait vraisemblablement au contentieux un acte qui ne respecterait pas ses prérogatives. Mais ici, l'article $299 \S 2$ ne mentionne que des «conditions d'accès aux fonds structurels » qui ne relèvent pas nécessairement des règlements relatifs à chacun d'entre eux : pour l'inscription des régions ultramarines parmi celles où l'intensité des financements communautaires est plus élevée (classement parmi les régions dites «objectif $\left.n^{\circ} 1 »\right)$, le règlement posant les règles les plus générales du droit dérivé de la Cohésion économique et sociale concerne l'ensemble des différents Fonds structurels et découle de la volonté unanime du Conseil, assortie de l'avis conforme du Parlement - selon l'article 161 (ex-art. 130 D) CE - et il est remarquable que son auteur les ait lui-même directement désignées aux côtés de certaines régions septentrionales de l'Europe, au lieu d'en confier la tâche à la

37. Règl. $\mathrm{n}^{\circ} 2395 / 79$ du Conseil du 30 octobre 1979 relatif au concours du FEOGA-section « orientation », concernant une action d'urgence pour la reconstitution des zones agricoles des départements français d'outre-mer endommagées par les cyclones « David » et « Frédéric », JOCE L 275, $1^{\text {er }}$ novembre 1979, p. 1

38. Voir par exemple, Claude Blumann, Politique agricole commune, Litec, 1996, $\mathrm{n}^{\circ}$ 604, p. 340. Cette tendance s'accentue encore pour la période 2000-2006 : Règl. $\mathrm{n}^{\circ} 1257$ du Conseil, 17 mai 1999, concernant le soutien au développement rural par le FEOGA..., art $35 \S 2,2^{\mathrm{e}}$ tiret (JOCE L 160, 26 juin 1999, p. 80). 
Commission, comme c'est le cas le plus général ${ }^{39}$, ou de se réserver la compétence d'exécution sur ce point, comme le lui permet l'article 202 (ex-art.145) CE. Si au-delà de l'aspect des montants disponibles, il convenait d'entendre l'expression «conditions d'accès » sous l'angle des difficultés administratives rencontrées pour obtenir des décisions favorables de concours, la seule intervention d'actes communautaires se révélerait insuffisante: le principe de subsidiarité inscrit dans le droit primaire [article 5 (ex-art. $3 \mathrm{~B}$ ) alinéa $2 \mathrm{CE}$ ], renforcé par le principe de partenariat repris dans le règlement portant dispositions générales sur les Fonds structurels pour la période 2000-2006 ${ }^{40}$, empêche l'atteinte à l'autonomie institutionnelle et administrative de chaque État membre que constituerait une gestion communautaire directe, même spécialement pour les régions ultramarines.

De façon plus globale, l'on sait que la révision par le traité d'Amsterdam a gommé la distinction matérielle figurant précédemment à l'ex-article $227 \S 2$; mais du point de vue procédural, resurgit la frontière de droit commun entre les domaines qui appellent des actes de premier niveau pris en forme plus solennelle et ceux qui peuvent être régis par des actes issus d'une procédure aussi simplifiée que celle mentionnée à l'article 299 § 2 alinéa 2 ; dans le premier cas, quel peut être le champ d'intervention de cette dernière procédure ? La question de l'élaboration des règlements relatifs aux différents fonds à finalité structurelle, en application de dispositions plus générales, illustre justement l'hypothèse où le traité impose une procédure pour l'adoption des actes de deuxième niveau. On peut alors se demander si la composante procédurale de l'article $299 \S 2$ ne pose pas une règle du même type.

En effet, dans le texte du traité révisé, l'alinéa 2 du nouvel article 299 § 2 affirme que les «mesures spécifiques » à arrêter par le Conseil à la majorité qualifiée visent à fixer des conditions de l'application du traité ; elles doivent contribuer à les préciser, les mettre en œuvre comme tout acte de droit dérivé au regard du droit

39. Règl. $n^{\circ}$ 1260/1999 du Conseil, 21 juin 1999, portant dispositions générales sur les Fonds structurels (précité), article 3 ; voir respectivement $\S 1^{\text {er }}$, al. 2 et $\S 2$ al. $1^{\mathrm{er}}$.

40. Règl. $n^{\circ} 1260 / 1999$ précité, art. $8 \S 1^{\mathrm{er}}$. 
primaire; mais le traité prévoit lui-même les conditions de son application, pour les régions ultramarines comme pour toute autre zone de l'espace communautaire. Par comparaison, l'article $227 \S 2$ alinéa 2 initial avait prévu que ces conditions étaient déterminées par le Conseil statuant à l'unanimité, semblant ouvrir la voie à l'énoncé par ce dernier de conditions dérogatoires par rapport au traité, avant l'intervention de la Cour de Justice dans un sens restrictif ${ }^{41}$. Cette modification de rédaction et le changement de procédure permettent de proposer une interprétation préservant l'effet utile de la nouvelle stipulation mettant à la charge du Conseil la mission d'élaborer ces dispositifs particuliers.

L'hypothèse avancée est la suivante : un acte de droit dérivé de premier niveau relatif à un secteur donné de l'activité communautaire est élaboré par le législateur communautaire, selon la procédure imposée par la base juridique pertinente de droit commun (par exemple la co-décision du Conseil et du Parlement, après consultation du Comité économique et social, du Comité des régions); son champ d'application territorial est celui de la Communauté elle-même, mais il renvoie à des actes de mise en œuvre la fixation de conditions particulières qui peuvent être différentes selon les régions ; en ce qui concerne les régions ultrapériphériques, comme il s'agit de tenir compte de situations reconnues atypiques par le traité, l'élaboration de ces actes échappe à la compétence d'exécution de la Commission et revient au Conseil qui statue cependant sur proposition de celle-ci et après consultation du Parlement européen, ce qui va dans le sens des efforts de ce dernier en vue d'augmenter son influence dans le processus d'élaboration des actes de deuxième niveau.

Par rapport au système du traité, ce schéma n'est pas vraiment exceptionnel. En effet, il est prévu que le Conseil «peu... se réserver, dans des cas spécifiques, d'exercer directement des compétences d'exécution » [article 202 (ex-art. 145) $3^{\mathrm{e}}$ tiret, $3^{\mathrm{e}}$ phrase CE]. Surtout, on remarque que, depuis les révisions effectuées à partir de l'Acte unique, dans certains cas le traité prédétermine la compétence du Conseil au stade des actes de mise en œuvre, assortie de l'obligation de procéder à certaines consultations. Par exemple, dans le cas des

41. CJCE, 19 février 1998, Chevassus-Marche, aff. C-212/96, Rec. p. I-743. 
règlements relatifs à la «Cohésion économique et sociale », après des dispositions générales relatives aux missions, à l'efficacité, à la coordination des fonds à finalité structurelle ${ }^{42}$, les «décisions d'application » relatives au Feder (art. 162 al. $1^{\text {er }} \mathrm{CE}$ ), de même que celles concernant le FEOGA et le FSE (art. 162 al. 2 CE), connaissent les procédures d'élaboration précitées imposées par le traité luimême ${ }^{43}$.

Dans le cas précis de l'article 299 § 2 alinéa 2 CE, la procédure qui y est prescrite peut donc être valablement utilisée, à chaque fois que des actes arrêtés selon des procédures de droit commun, en général plus solennelles, renvoient à des actes de mise en œuvre concernant spécialement les régions ultrapériphériques. Mais au-delà de la confirmation de la licéité de la compétence retenue du Conseil, selon

42. Sur la base de l'art. 130 D CEE initial (issu de l'Acte unique), les institutions communautaires ont opté pour deux règlements successifs élaborés selon la même procédure [V. respectivement le règl. $n^{\circ}$ 2052/88 du Conseil du 24 juin 1988 (fréquemment dénommé « règlement-cadre »), JOCE L 185, 15 juill. 1988, p. 9, modifié par le règl. $\mathrm{n}^{\circ}$ 2081/93 du 20 juill. 1993, JOCE L 193, 31 juill. 1993, p. 5 ; et le règl. $\mathrm{n}^{\circ}$ 4253/88 du Conseil du 19 déc. 1988 (fréquemment dénommé «règlementcoordination »), JOCE L 374, 31 déc. 1988, p. 1 et s., modifié par le règl. $\mathrm{n}^{\circ}$ 2082/93 du 20 juillet 1993, JOCE L 193, 31 juill. 1993, p. 20 et s.] avant que la Commission ne propose un unique règlement portant dispositions générales pour la période 2000-2006 [Proposition (98/0090 AVC) in document COM (1998) 131 final, 18 mars 1998 ; JOCE C 176, 9 juin 1998, p. 1 \& s.], alors que la rédaction de l'article $130 \mathrm{D}$ CE issue du traité de Maastricht incitait plutôt au maintien de deux règlements distincts [Voir actuel article 161 CE et règl. $n^{\circ} 1260 / 1999$ précité].

43. On peut trouver d'autres illustrations concernant la fixation par le traité de l'autorité compétente et de la procédure pour les actes de deuxième niveau, en matière d'environnement [pour la mise en œuvre des programmes d'action à caractère général, l'art. 175 al. 2 CE (ex-art. $130 \mathrm{~S}$ ) prévoit la compétence du Conseil, mais selon une procédure variable : soit celle de co-décision, assortie de la consultation du Comité économique et social et du Comité des régions, soit l'exigence de l'unanimité au Conseil, après consultation du Parlement, du Comité économique et social et du Comité des régions... (art. 172 al. $2 \mathrm{CE}$ )], en matière de recherche et de développement technologique [pour les programmes spécifiques mettant en œuvre un programme-cadre, l'art. 166 \& CE (ex-art. 130 I) impose la compétence du Conseil statuant à la majorité qualifiée, après consultation du Parlement et du Comité économique et social (voir aussi nouvel art. 172 al. $1^{\text {er }} \mathrm{CE}$ ) ; dans d'autres cas, est exigée la co-décision et la consultation du Comité économique et social (art. 172 al. 2 $\mathrm{CE})]$. 
l'article $202 \mathrm{CE}$, cette stipulation constitue-t-elle une clause générale d'habilitation du Conseil qu'il ne pourrait, même en accord avec le Parlement européen, transgresser ? Il en serait ainsi si la frontière entre les actes de premier et de deuxième niveaux était véritablement nettement déterminée, si l'on pouvait identifier une ligne de démarcation rendant obligatoire leur dissociation. Or le législateur communautaire peut préférer fixer lui-même dans un acte de premier niveau, c'est-à-dire selon une procédure généralement plus complexe, des lignes directrices pour la mise en œuvre, au lieu de renvoyer à un acte ultérieur d'application pris selon une procédure simplifiée, émanant soit du Conseil, soit de la Commission. Tant que l'on demeure dans la logique de l'exploitation des potentialités du droit commun en vue d'une adéquation des normes à la situation des régions ultramarines, le choix du renvoi à un acte communautaire d'exécution, celui de l'autorité compétente pour l'arrêter et celui de sa procédure d'élaboration relèvent davantage de l'opportunité que de prescriptions juridiques. Au mieux, la règle de procédure figurant à l'article 299 § 2 alinéa 2 se présente comme une très forte incitation à confier la compétence d'exécution au Conseil, plutôt qu'à la Commission, même si celle-ci peut se voir habilitée à prendre des actes d'application à une étape ultérieure.

Ainsi, à ce stade, le résultat de la révision ne se situe sans doute pas à la hauteur des espérances de ceux qui l'ont revendiquée : le fait d'avoir réussi à faire inscrire des contraintes procédurales minimales la consultation du Comité des régions n'étant même pas prévue -, en vue d'obtenir plus facilement un degré de différenciation juridique supérieur, se heurte aux exigences de la réaffirmation du principe d'intégration dès l'alinéa $1^{\text {er }}$ du $\$ 2$ du nouvel article 299 CE, d'autant que l'alinéa 4 impose de veiller à l'intégrité de l'ordre juridique communautaire. Même si les régions ultramarines doivent au moins pouvoir bénéficier de ce qui est permis à l'égard des régions européennes des États membres, l'effet utile de la dimension procédurale de l'article $299 \S 2$ se révèle faible dans cette modalité de conciliation de la différenciation et de l'intégration.

L'insistance des rédacteurs de l'article 299 § 2 sur la légitimité de mesures dites «spécifiques » est telle qu'il convient d'étudier la 
question de la licéité d'un degré supplémentaire de différenciation qui serait propre aux régions ultramarines, étant entendu que l'on doit interpréter restrictivement les possibilités d'altération de la portée des normes d'application générale.

\section{II. - UNE DIFFERENCIATION PROPRE AU STATUT DE REGIONS ULTRAPERIPHERIQUES ?}

La présence même du $\S 2$ dans l'article 299 incite à supposer la virtualité d'une différenciation supérieure à celle tolérée pour les régions exclusivement concernées par le $\S 1^{\text {er }}$, alors que celui-là rappelle le principe d'intégration déjà impliqué par celui-ci. Mais une fois admise la licéité d'un degré supérieur d'altération de l'orthodoxie juridique communautaire (A), subsisteraient des doutes sur le recours à la procédure simplifiée figurant à son deuxième alinéa pour leur élaboration (B).

\section{A. - Le degré tolérable de différenciation accrue}

Bien que l'alinéa 2 de l'article 299 § 2 mette l'accent sur la légitimité de la différenciation en faveur des régions ultrapériphériques, il n'est pas pour autant certain que des actes actualisant une potentialité de divergence par rapport au droit commun entrent dans la catégorie juridique des dérogations.

\section{1. - La légitimité d'un degré supérieur de différenciation}

La légitimité de normes présentant un degré plus élevé de différenciation en faveur des régions ultramarines étant affirmée de façon insistante à l'alinéa 2 de l'article 299 § 2, il y a tout lieu de penser que les auteurs de tels actes devraient les motiver en considération de difficultés propres à ces régions - «les caractéristiques et contraintes particulières » (al. 4) ; «les facteurs dont 
la combinaison et la permanence » aggravent leur situation (al. 2) -, et non seulement de celles qui se rencontrent à une moindre intensité dans des zones européennes de la Communauté. A cette ambition de traiter la situation atypique des diverses régions ultrapériphériques, y compris pour compenser des désavantages irréductibles, doivent correspondre des contenus originaux par rapport à ceux en vigueur dans les régions européennes et non transposables dans celles-ci. Ils peuvent alors se situer au-delà de la démarche d'exploitation maximale des possibilités offertes par le droit commun, celui-ci étant présenté comme inadéquat à la situation à régir.

Mais il s'agit aussi, pour ces régions ayant fait l'objet d'une réaffirmation solennelle de leur pleine intégration dans la Communauté, de montrer qu'un écart important par rapport à la stricte rigueur communautaire ne porte pas fondamentalement atteinte aux intérêts de la Communauté ${ }^{44}$. La balance d'intérêts peut, pour ces régions particulières - et de peu de poids dans l'ensemble communautaire -, se situer à un point d'équilibre différent de celui qui serait admis pour l'ensemble des régions implicitement concernées par le $\S 1^{\text {er }}$ de l'article 299, sans affecter fondamentalement l'ordre juridique communautaire.

On en trouve l'indice dans la nouvelle formulation choisie pour exprimer cette idée au niveau du droit primaire : selon l'alinéa 4 de l'article 299 § 2, il s'agit, s’il est jugé approprié d'exploiter la possibilité d'arrêter des dispositifs différenciés, de le faire «sans nuire à... l'ordre juridique communautaire », alors que les expressions habituellement rencontrées parlaient d'éviter une altération dans «une mesure contraire à l'intérêt commun ${ }^{45}$. De cette modification de

44. Sur la nécessaire sauvegarde des intérêts de la Communauté, voir par exemple : CJCE, 9 novembre 1995, Atlanta Fruchthandelsgesellschaft mbH e. a., aff. C-465/93, point 43, Rec. p. I-3799.

45. Voir par exemple la décision (89/688) du Conseil du 22 décembre 1989 sur l'octroi de mer (JOCE L 399, 31 décembre 1989, p. 46.), art. 2 § 3: «sans être pour autant de nature à altérer les conditions des échanges dans une mesure contraire à l'intérêt commun»; également les articles 36, 85, 86, 100 A $\S 4$ du traité CE (numérotation antérieure au traité d'Amsterdam) : interdiction de « restriction déguisée au commerce entre États »; obligation de ne pas «affecter le commerce entre États ». 
vocabulaire, figurant spécialement dans une stipulation relative à l'outre-mer, on peut déduire la licéité d'une différenciation supérieure sans qu'une «nuisance » en découle, surtout si des précautions sont prises: par exemple, le champ d'application circonscrit devrait permettre d'en soutenir le caractère non attentatoire à la construction communautaire, dans le respect du principe de proportionnalité ${ }^{46}$.

Dans cette perspective, se confirme l'existence d'un statut particulier pour les régions visées par l'article 299 § , simultanément objet d'une réaffirmation de leur pleine intégration juridique dans la Communauté. Pour autant, sachant que ceux qui ont revendiqué la modification du traité à ce sujet cherchaient à supprimer toutes restrictions aux dérogations possibles, le résultat risque de ne pas se situer à la hauteur de leurs espérances.

\section{2. - La qualification juridique de la différenciation tolérée}

La qualification juridique de la différenciation tolérée par ce texte prioritairement marqué du sceau de l'intégration semble ne pas devoir être celle de la dérogation au droit primaire. En effet, outre le rappel à la préservation de " l'intégrité et à la cohérence de l'ordre juridique communautaire, y compris le marché intérieur et les politiques communes », l'article $299 \$ 2$ ne comporte pas expressément d'habilitation à déroger au traité lui-même. La situation est donc différente des cas où existe « une possibilité de différenciation juridique inscrite dans la mesure communautaire ${ }^{47}$ elle-même. Et si les auteurs du droit dérivé, aiguillonnés par divers groupes d'intérêt ${ }^{48}$,

46. Voir par exemple, F. Picod, «Principe généraux de droit », in Ami Barav et Christian Philip, Dictionnaire juridique des Communautés européennes, P.U.F., 1993, p. 863 et s.

47. Denys Simon, «Articles $130 \mathrm{R}, \mathrm{S}, \mathrm{T}$ CE », in Vlad Constantinesco et autres : Traité sur l'Union Européenne - Commentaire article par article, Economica, 1995, p. 486.

48. Sur EURODOM et l'UPEC, v. Marc Janus, « Les départements français d'outre-mer, la République française et la Communauté Économique Européenne : Esquisse d'une trilogie partenariale », mémoire pour le DEA Administration Publique 
interprétaient leur tâche d'élaborer des «mesures spécifiques visant... à fixer les conditions de l'application du... traité », comme une habilitation à y déroger, il y aurait lieu de craindre une sanction contentieuse de la Cour tout autant tenue de défendre «l'intégrité et... la cohérence de l'ordre juridique communautaire ». Au lieu de s'en remettre à son oracle pour qu'elle livre ses réponses au fur et à mesure des saisines, il semble préférable de rechercher dans sa jurisprudence des éléments d'élucidation de la nature juridique et du régime des écarts tolérables par rapport à la stricte orthodoxie communautaire.

L'on se souvient en effet que sous l'empire de la rédaction initiale de l'article $227 \S 2$, les stipulations du traité évoquées par son alinéa 1er étaient présentées comme intangibles, alors qu'une plus grande souplesse était permise pour les autres (alinéa 2). Dans la ligne de l'arrêt Hansen, les auteurs du droit dérivé relatif aux DOM ont interprété «les plus larges possibilités de prévoir des dispositions particulières, adaptées aux exigences spécifiques de ces parties du territoire français ${ }^{49}$ comme une autorisation d'adopter des actes de droit dérivé s'écartant des règles communes prévues en droit primaire et ont parlé de dérogation ${ }^{50}$. Si l'on peut comprendre ce terme comme signifiant une divergence de contenu entre deux normes, soit de même rang - en ce sens la règle spéciale déroge à la règle de portée générale

Outre-Mer, Université Antilles-Guyane, CRPLC, Schœlcher, 1993 (Publication : Ed. L'Harmattan, Paris, 1995, p. 53 et s.) ; «EURODOM - un lobby original des régions ultrapériphériques de la Communauté européenne », Revue du Marché commun et de l'Union européenne, $\mathrm{n}^{\circ} 388$, mai 1995, p. 326 et $\mathrm{s}$.

49. CJCE, 10 octobre 1978, aff. 148/77, Rec. p. 1787, point 9. Ce passage est encore cité en 1998 : CJCE, 19 février 1998, Chevassus-Marche, aff. C-212/96, Rec. p. I-743, point 32.

50. Par exemple en matière fiscale, à propos du rhum traditionnel des DOM, les autorités communautaires se sont résolues à deux reprises à autoriser l'État membre à taxer moins lourdement ce produit, de façon dérogatoire à l'article $95 \mathrm{CE}$ initial, admettant ainsi que la prise en considération de la provenance de cette marchandise conduisait à tolérer une imposition discriminatoire ou indirectement protectrice : Décision du Conseil (88/245) du 19 avril 1988, JOCE L 106, 27 avril 1988, p. 33 ; Décision du Conseil du 30 octobre 1995 (non publiée ; texte reproduit in E. Jos et D. Perrot, Les départements d'outre-mer et le droit de l'Union européenne - Les grands textes, Paris, La Documentation française, coll. Travaux de la CEDECE, 2000, p. 168). 
- soit de valeurs juridiques différentes - la disposition de droit dérivé diverge de la stipulation de droit primaire, parce que celui-ci en a fourni la base légale -, la jurisprudence récente est venue rappeler que si le statut de ces régions ultramarines tolère de tels écarts, ce n'est pas au titre d'une dérogation au premier sens du terme. A la différence des régions citées au paragraphe 5, selon le traité alors en vigueur (visées aux paragraphes 5 et 6 depuis le $1^{\text {er }}$ mai 1999), leur insertion dans le cadre juridique communautaire est marquée par la pleine intégration et le droit primaire ne comporte, à leur propos, aucune autorisation expresse à maintenir ou à instituer des dérogations.

Des affaires sont en effet pendantes devant la Cour lorsque la question du statut des régions ultrapériphériques est véritablement traitée par la Conférence intergouvernementale; elles posent la question de la validité de l'acte du Conseil autorisant l'État membre à maintenir la perception de l'octroi de mer - précédemment qualifié de «taxe d'effet équivalent» strictement interdite par le droit communautaire primaire ${ }^{51}$, moyennant une réforme du droit interne : la décision 89/688 du Conseil du 22 décembre 1989 vise à le transformer en imposition intérieure, mais le Conseil a conscience qu'il ne remplira pleinement les critères de licéité posés par l'article 95 du traité (à présent, article $90 \mathrm{CE}$ ) que lorsque le régime temporaire des exonérations au profit de la production locale aura pris fin ${ }^{52} \mathrm{Au}$ moment où la Cour statue sur la validité de la décision du Conseil tolérant cette entorse au principe du caractère non discriminatoire et non protecteur des impositions intérieures licites, l'avocat général a rendu des conclusions contraires depuis onze mois ${ }^{53}$, et la teneur de la

51. V. CJCE, 16 juillet 1992, C-163/90, Legros et autres, Rec. p. I-4625, particulièrement points 16 à 18 ; voir aussi : CJCE, 9 août 1994, C-363/93, Lancry, Rec. p. I-3957.

52. Décision (89/688) du Conseil du 22 décembre 1989, $9^{\mathrm{e}}$ considérant : « au terme de cette période, le régime fiscal devrait donc en principe être pleinement conforme aux principes de l'article 95 du traité », JOCE L 399, 31 décembre 1989, p. 46.

53. Alors que les travaux de la Conférence intergouvernementale piétinent sur la future rédaction de l'article 227 § 2, l'avocat général M. Tesauro conclut à l'invalidité, au motif de l'impossibilité de s'écarter des règles de la libre circulation des marchandises dont l'article 95 alors en vigueur, constitue un complément, voire un accessoire ; il se prononce sur la licéité des aides publiques internes, sous la condition 
révision issue du traité d'Amsterdam est pour l'essentiel connue depuis huit mois. L'invocation par la Cour d'une stipulation du traité encore en vigueur - son article 226 -, non seulement considérée comme obsolète, mais en voie d'abrogation formelle ${ }^{54}$, peut inciter à considérer que la solution alors dégagée se trouve dépourvue de tout fondement sous l'empire du traité révisé ; toutefois elle comporte des éléments permettant d'augurer la démarche jurisprudentielle ultérieure.

La volonté de la Cour en formation plénière est en effet que la portée de son raisonnement dépasse le cadre strict des points soulevés par les juges de renvoi. Ainsi, par-delà la confrontation de la décision du Conseil aux exigences des articles du traité concernant la libre circulation des produits, la Cour aborde la question de la libre circulation des personnes, pour affirmer : «il ne saurait être admis que la jouissance, par les ressortissants communautaires, des droits consacrés par les dispositions du traité... droits faisant partie de leur patrimoine juridique, soit susceptible d'être altérée, au fil du temps, par des décisions du Conseil $»^{55}$. Elle en conclut que, comme pour les

« de respecter les procédures prévues par le traité » [Conclusions du 20 mars 1997, dans les affaires jointes C-37/96 et C-38/96, Sodiprem SARL e. a. et Roger Albert SA (CJCE, 30 avril 1998, Rec. p. I-2039 et s.), et dans l'affaire C-212/96, Paul ChevassusMarche (arrêt précité du 19 février 1998), particulièrement points 27 et s. et point 34]. Cela a vraisemblablement poussé les milieux intéressés au maintien du système protecteur à se mobiliser pour obtenir que le texte du traité effectivement adopté en juin 1997 comporte la mention : «Le Conseil... tient compte des domaines tels que les politiques douanières..., la politique fiscale... » et aussi « les aides d'État » (alinéa 3 du nouvel article 299 § 2 CE).

54. Pour conclure à la validité de la décision $89 / 688$, la Cour se réfère à l'article 226 du traité initial, clause applicable «au cours de la période de transition » échue depuis 1970 et s'appuie sur le renvoi par l'article $227 \S 2$ alinéa 3 aux procédures prévues par le traité, et notamment à celle de l'article 226 [CJCE, 19 février 1998, Paul Chevassus-Marche, précité, point 41. «S'il est vrai que l'article 226 du traité ne s'appliquait, selon ses propres termes, que pendant la période de transition, il y a lieu d'observer que l'article 227, paragraphe 2, troisième alinéa, du traité renvoie aux procédures prévues à cet article »]. Le traité d'Amsterdam déclare l'abroger, à son article 6, point 75 (Union européenne, Traité d'Amsterdam, Luxembourg, OPOCE, 1997, p. 66).

55. Arrêt précité, point 42. Lorsqu'elle parle «des travailleurs et d'autres personnes physiques et morales », elle ne rappelle pas que la libre prestation de service (relevant de l'alinéa $1^{\mathrm{er}}$ de l'article $227 \S 2 \mathrm{CE}$ alors en vigueur) doit être distinguée 
droits des personnes, en matière d'impositions frappant les marchandises, le législateur communautaire est soumis à l'observation de certaines conditions qui se trouvent réunies en l'espèce. Ainsi, le Conseil n'aurait pu «en tout état de cause, autoriser un système d'exonération d'ordre général ou systématique susceptible d'aboutir à la réintroduction d'une taxe équivalant à un droit de douane $»^{56}$.

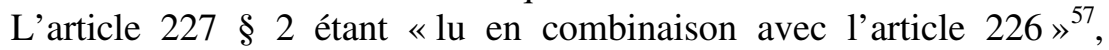
l'assouplissement temporaire de la règle générale des impositions intérieures indifférenciées ne peut, même pour les DOM, régions juridiquement intégrées à la Communauté, relever que du régime des mesures de sauvegarde encadrées par les limites exprimées par l'article 226 initial et transposables au-delà de son champ propre.

On peut admettre que pour des autorités locales ou des opérateurs économiques intéressés à obtenir des atténuations de la rigueur du droit commun, peu importe le caractère de dérogation ou de mesure de sauvegarde attribué à une disposition allant dans le sens de leurs aspirations. Mais si des traductions concrètes peuvent être assez voisines, les conséquences juridiques, selon la qualification retenue, sont différentes.

Dans le cas des dérogations prévues par le droit primaire, il n'est pas obligatoire de soumettre l'État bénéficiaire à un «contrôle communautaire » autre que le contrôle juridictionnel, même si l'autorisation donnée par le législateur communautaire à l'État de déroger peut s'accompagner de la mission confiée à la Commission de vérifier la persistance des raisons de l'octroi de la dérogation et de

des autres formes de libre circulation des personnes, mais insiste sur la nécessaire préservation du patrimoine juridique des particuliers, même lorsque le législateur communautaire agit dans des domaines (relevant de l'alinéa 2 de l'article 227 § 2) où sa latitude est plus large.

56. Arrêt précité, point 37 ; v. aussi, point 45. Sur la déclaration d'invalidité de l'article 4 de la même décision (89/688) qui autorisait l'État membre à maintenir pendant trois ans le régime antérieur de l'octroi de mer. V. CJCE, 9 août 1994, Lancry, C-363/93, précité, point 38.

57. Arrêt précité, point 53 ; voir aussi point 52 : « conditions strictes... interprétées à la lumière des limites prévues à l'article 226 ». 
formuler des propositions en conséquence ${ }^{58}$. En revanche, la mise en œuvre d'une clause de sauvegarde exige non seulement des garanties procédurales - l'autorisation préalable donnée à l'État par une institution communautaire, un contrôle communautaire étroit sur le bénéficiaire - mais aussi un contenu encadré ${ }^{59}$, car la mise à l'écart ponctuelle de la stricte orthodoxie juridique communautaire ne signifie pas suspension de l'état de droit, selon l'expression du Doyen Boulouis $^{60}$, le respect des principes généraux du droit continuant notamment à s'imposer ${ }^{61}$.

Aussi, lorsque le Conseil a permis, par la décision 89/688 du 22 décembre 1989, des mesures «nécessaires, proportionnelles et précisément déterminées ${ }^{62}$, assorties d'un encadrement suffisant de l'usage qu'en feraient les autorités internes, il s'est situé dans la ligne des contraintes de l'article $226 \mathrm{CE}$, c'est à dire des «conditions strictes », tenant tant au fond - adéquation à l'objectif de développement économique et social, respect du principe de proportionnalité - qu'aux procédures de contrôle communautaire mises sur pied pour vérifier le respect des premières - notification préalable à la Commission lui permettant d'exercer «un contrôle strict », rapport

58. Directive 94/80 précitée sur le droit de vote aux élections municipales, art 12 $\S 4$.

59. Sur les principes à caractère général déduits par la jurisprudence de l'article 226 pour l'ensemble des clauses de sauvegarde, v. J. Boulouis, R.-M. Chevallier : Grands arrêts de la CJCE, Tome 1, Dalloz, 1994, p. 144 et s. ; L. Cartou, "Article 226 », in Vlad Constantinesco et autres (dir.), Traité instituant la CEE - Commentaire article par article, Economica, 1992, p. 1415 ; Y. Gautier, "Clauses de sauvegarde », in A. Barav et C. Philip, Dictionnaire juridique des Communautés européennes, PUF, 1993, p. 169.

60. Observations sous CJCE, 13 mai 1971, « International Fruit, aff. 41 à 44/70 », in Grands arrêts de la CJCE, Tome 1, Dalloz, 1994, p. 146.

61. Sur la confrontation d'un règlement instaurant des mesures de sauvegarde aux principes de sécurité juridique et de proportionnalité, voir CJCE, 7 févr. 1973, Schroder, aff. 40/72, points 15 et s, Rec. p. 125.

62. Arrêt Chevassus-Marche précité, point 49 ; arrêt Sodiprem SARL e. a., aff. C-37 \& 38/96 précité, point 35. Voir aussi, CJCE, ordonnance du 7 juill. 1998, Société Béton Express e. a., aff. C-405 à C-408/96, Rec. p. I-4253 et s. 
de cette dernière sur l'incidence sur la libre circulation des marchandises $-{ }^{63}$.

Le rappel par la Cour de Justice des exigences de l'article 226 $\mathrm{CE}$ initial en voie d'abrogation, au moment où l'entrée en vigueur du traité d'Amsterdam dépend encore de nombreuses ratifications, permet sans doute d'éclairer l'obligation faite par le futur article 299 § 2 CE de ne pas nuire «à l'intégrité et à cohérence de l'ordre juridique communautaire, y compris le marché intérieur... ». En effet, les lignes directrices de l'article 226 primitif ont inspiré de nouvelles stipulations du traité lors de révisions précédentes, comme les articles $8 \mathrm{C}$ et $100 \mathrm{~A}$ CEE ajoutés par l'Acte unique ${ }^{64}$, ou encore l'article 130 R CE modifié par le traité de Maastricht ${ }^{65}$. Il en a aussi été tenu compte dans la décision du Conseil relative aux compétences d'exécution conférées à la Commission: lorsqu'il lui revient de décider des mesures de sauvegarde en application d'une clause prévue en droit dérivé, à défaut de précisions contraires dans l'acte de premier niveau, s'applique une procédure spéciale ${ }^{66}$.

La disparition de l'ex-article 226 du traité révisé n'entraîne ni celle des principes de portée générale qu'il comportait, ni celle de l'exarticle $7 \mathrm{C}$, devenu article $15 \mathrm{CE}$ - initialement introduit dans le traité

\section{Arrêt précité, points 47 à 51 .}

64. Sur l'article $8 \mathrm{C}$ al. 2 CEE (il devient l'art. $7 \mathrm{C} \mathrm{CE}$ après le traité de Maastricht, puis l'art. $15 \mathrm{CE}$ après le traité d'Amsterdam), voir L. Cartou, «Article 226 », et D. Simon, «Article $8 \mathrm{C}$ », in Vlad Constantinesco et autres (dir.), Traité instituant la CEE - Commentaire article par article, Economica, 1992, p. 1416 et p. 82. Sur la procédure communautaire de contrôle en application de l'article $100 \mathrm{~A} \S 4$ et $\S 5$ CEE (que l'on retrouve aux $\S 4$ à 7 et au $\S 10$ du nouvel art. $95 \mathrm{CE}$ ), voir D. Simon, "Article $100 \mathrm{~A}$ », op. cit. p. 568 et s. En ce sens, la rédaction du nouvel article $95 \mathrm{CE}$ est plus claire : la Commission peut expressément rejeter les dispositions nationales ( $\$ 6$ ); en cas d'approbation, l'État est « autorisé » à les maintenir (\$7).

65. Sur l'article $130 \mathrm{R} \mathrm{CE}$ et la clause de sauvegarde insérée au $\S 2$ alinéa 2 (nouvel art. $174 \mathrm{CE}$ après le traité d'Amsterdam), v. D. Simon, «Articles $130 \mathrm{R}, \mathrm{S}, \mathrm{T}$ $\mathrm{CE}$ », in Vlad Constantinesco et autres: Traité sur l'Union Européenne Commentaire article par article, Economica, 1995, p. 485.

66. Décision (87/373) du Conseil du 13 juillet 1987 (dite «comitologie ») : art. 3 [JOCE L 197, 18 juill. 1987, p. 33], modifiée et remplacée par la décision (1999/468) du Conseil du 28 juin 1999 : art. 6 [JOCE L 184, 17 juill. 1999, p. 23]. 
CEE par l'Acte unique sous le numéro $8 \mathrm{C}$ - relatif au marché intérieur. Imposant de tenir compte de «l'ampleur de l'effort que certaines économies présentant des différences de développement devront supporter au cours de la période d'établissement du marché intérieur », il prévoit même que les dispositions du droit dérivé peuvent prendre « la forme de dérogations », mais sans préciser la possibilité de déroger au traité lui-même; en revanche, le rappel des limites classiques en matière de clauses de sauvegarde a conduit à ranger cette «reconnaissance d'un assouplissement indispensable du principe d'unicité du droit communautaire ${ }^{67}$ dans cette dernière catégorie.

Ainsi, si le nouvel article 299 § 2 CE incite, du fait d'une abondante motivation de la légitimité de mesures différenciées, à une conciliation moins rigoriste que pour les zones européennes en difficulté entre, d'une part, les exigences de l'unité du marché et de l'uniformité du droit communautaire ${ }^{68}$ et, d'autre part, le besoin de remédier aux handicaps de ces régions ou de les compenser, il convient de les assortir de conditions rappelant la révérence due au système général du traité. Peut-on alors se satisfaire d'une procédure d'adoption aussi souple que celle qui figure à l'article 299 § $2 \mathrm{CE}$, pour des actes autorisant temporairement des États à suspendre l'accomplissement de certaines de leurs obligations communautaires?

\section{B. - Le recours à une procédure simplifiée?}

Il est difficile d'admettre que la procédure d'élaboration d'un acte de droit dérivé restreignant la portée d'une clause du traité d'application générale soit plus simple que lorsqu'il s'agit de lui donner plein effet ; cela conduit à explorer la question du recours à une base juridique cumulative.

67. D. SIMON, Article 8 C, op. cit., p. 82.

68. Sur la préservation de ces exigences, dans le cadre de mesures de sauvegarde à destination des DOM, régions en difficulté, voir D. Simon, «Article 8 C», op. cit., p. 83 . 


\section{1. - Les doutes relatifs à la licéité du simple respect de la procédure mentionnée}

Alors que la mise en œuvre des clauses de portée générale du traité, permettant au législateur communautaire d'écarter la pleine expression $\mathrm{du}$ principe d'intégration, impose le respect d'une procédure plus solennelle que celle qui est prévue à l'article 299 § 2 $\mathrm{CE}$, il y a tout lieu de craindre qu'un acte communautaire fondé sur ce seul article, pour faire échapper les régions ultrapériphériques aux rigueurs du droit commun, constitue une atteinte à l'intégrité de l'ordre juridique communautaire. Celle-ci devant être sauvegardée tout particulièrement en ce qui concerne le marché intérieur (et les politiques communes) selon les termes de l'alinéa 4 de ce même article, les hypothèses où le Conseil statue seul à la majorité qualifiée se rencontrent de plus en plus rarement dans les bases juridiques auxquelles renvoient les articles 14 et $15 \mathrm{CE}$, même quand il s'agit de prévoir des dispositifs non différenciés dans des actes de premier niveau. Si, dans le cas des régions ultrapériphériques (ou celui d'autres zones de la Communauté), il était jugé utile d'exploiter l'habilitation figurant à l'article 15 alinéa 2, dans la mesure permise par lui « caractère temporaire », apportant « le moins de perturbation possible au fonctionnement du marché commun »-, l'autorisation de suspendre le plein effet d'une stipulation de droit primaire ne semble pas pouvoir être donnée par un acte de droit dérivé de premier niveau pris selon une procédure moins solennelle que celle qui est de règle pour l'élaboration de cet acte lui-même [comme dans nombre de cas évoqués dans la Première partie de cette étude, lorsque le traité prescrit la co-décision du Parlement et du Conseil, voire exige cumulativement l'unanimité au sein de celui-ci (selon, par exemple, l'actuel article 47 $\S 2 \mathrm{CE})]$.

Il est en revanche de pratique courante qu'une clause de sauvegarde figure dans un acte de premier niveau lui-même élaboré selon la procédure prévue dans la base juridique de droit commun ; en cas d'application, il ne s'agit que d'altérer la portée d'un acte de droit dérivé et il est habituel de confier le déclenchement de mesures de sauvegarde à la Commission. Pour ces dernières, la décision du Conseil relative aux compétences d'exécution conférées à la 
Commission retient une procédure d'élaboration particulière, applicable à défaut de précisions contraires dans l'acte de premier niveau : elle doit communiquer au Conseil et aux États membres ses décisions ; tout État peut les déférer au Conseil qui dispose, selon le cas, d'un pouvoir de réformation, de confirmation, ou d'abrogation, parfois implicite; et lorsqu'il statue explicitement, il le fait à la majorité qualifiée ${ }^{69}$. On peut donc admettre que la prescription de l'article 299 \$ 2 CE prévalant sur une décision de ce type du Conseil, il ne dispose pas de la compétence de confier l'adoption des mesures de sauvegarde à la Commission, mais doit arrêter lui-même les actes en cause. L'élaboration de ces décisions, intervenant en deuxième niveau, ne nécessite donc ni l'accord formel du Parlement dans le cadre de la co-décision, ni les avis du Comité économique et social et du Comité des régions, ni le vote unanime du Conseil.

Mais s'il s'agit, dans le cas particulier des régions ultrapériphériques, d'aller jusqu'à altérer la portée des stipulations du traité lui-même, sans que celui-ci l'ait expressément prévu, il serait sûrement opportun d'invoquer l'article 299 § 2 du point de vue de la légitimité de la décision à prendre; il serait également prudent de ne pas se limiter à la procédure qu'il prescrit et de recourir à une base juridique cumulative. Celle-ci pourrait-elle être trouvée dans le nouvel article $308 \mathrm{CE}$, qui reprend l'ex-article 235 initial, en vue d'une plus grande sécurité juridique ${ }^{70}$ ?

69. Décision (87/373) du Conseil du 13 juillet 1987 (dite «comitologie ») : art. 3 [JOCE L 197, 18 juill. 1987, p. 33], modifiée et remplacée par la décision (1999/468) du Conseil du 28 juin 1999 : art. 6, particulièrement point c) [JOCE L 184, 17 juill. 1999, p. 23].

70. Cet argument avait été retenu par la Cour : CJCE, 12 juillet 1973, MasseyFergusson, aff. 8/73, point 4, Rec. p. 897. Mais le «motif n'est plus réapparu dans la jurisprudence » ultérieure et il «n'implique pas que l'on valide à tout prix des actes viciés »: Anne Wachsmann, «Le contentieux de la base juridique dans la jurisprudence de la Cour», Europe, janvier 1993, p. 1 et s. Voir par exemple, sur l'octroi de mer, CJCE, 9 août 1994, Lancry, C-363/93, précité. 


\section{2. - L'utilité d'une double base juridique}

L'arrêt précité du 19 février 1998 rendu par la Cour en formation plénière peut encore à ce propos fournir des indications.

Certes, en fonction de la rédaction alors en vigueur de l'article $227 \S 2 \mathrm{CE}$ et de la «distinction fondamentale » qu'il opère entre les matières relevant de son alinéa 1er et les autres, la Cour rappelle que l'article $235 \mathrm{CE}$ ne permet pas de suspendre l'application des articles prohibant les taxes d'effet équivalent ${ }^{71}$; lorsque, en revanche, il s'agit d'« enfreindre l'article 95 » (point 25), «seul l'article 227 du traité éventuellement lu en combinaison avec l'article 235» serait susceptible d'en fournir la base juridique (point 27). La Cour n'argumente pas sur l'utilité ou le caractère superflu de cette base juridique cumulative. Devant apprécier la décision 89/688 dans le contexte juridique de son édiction - antérieure au traité sur l'Union elle n'a pas non plus estimé devoir sanctionner l'exigence de consultation obligatoire du Parlement européen impliquée par l'article 235, alors qu'elle n'est que facultative en application stricte de l'article $227 \S 2$; sans doute, ici «ne se trouvent pas déjouées... les règles du traité relatives à la formation de la volonté du Conseil ou à la répartition des pouvoirs entre institutions $»^{72}$.

Mais les orientations tracées par la Cour, alors que le texte du traité révisé est connu pour l'essentiel depuis juin 1997, peuvent éclairer sur la mise en œuvre du nouvel article 299 § 2 - qui insiste sur l'intégrité de l'ordre juridique communautaire -, lu en combinaison avec le nouvel article 308 (ex-article 235) qui permet aux institutions d'agir lorsque le traité n'a pas prévu les «pouvoirs d'action requis à cet effet ». En ce qui concerne l'autorisation temporaire de suspendre des obligations communautaires issues du droit primaire, au bénéfice des régions ultrapériphériques, cela amène à considérer que l'article

71. Arrêt précité (CJCE, 19 février 1998, C-212/96, Paul Chevassus-Marche) : son point 36 rappelle l'arrêt Lancry précité.

72. Arrêt Massey-Fergusson, précité, point 4. Voir René Milas, «La concurrence entre les bases légales des actes communautaires », Rev. du Marché Commun, $\mathrm{n}^{\circ} 289$, juil.-août 1985, p. 445 et s. 
$299 \S 2$ ne s'avère pas une base juridique suffisante, ce qui constitue un résultat inverse de celui recherché par ceux qui ont réclamé la révision du traité sur cette question précise. Surtout, le recours à l'article 308 comme base juridique cumulative exige que le Conseil statue à l'unanimité, les autres phases de procédure étant similaires (proposition de la Commission, consultation du Parlement).

D'un point de vue tactique, agir ainsi peut être une utile précaution ; cela contribuerait à prévenir des contestations émanant d'États qui, s'étant trouvés minoritaires lors d'un vote à la majorité qualifiée, prétendraient que, pour une atteinte à des règles juridiques essentielles de la construction communautaire et dans le souci de préserver « l'intégrité de l'ordre juridique communautaire », il faudrait recourir à une procédure plus consensuelle, sinon plus solennelle.

D'un point de vue juridique, il convient de rappeler que la Cour de justice peut admettre l'exigence d'une double base légale, lorsque se présentent des circonstances spéciales, malgré sa tendance à préférer une base juridique unique, celle qui permet l'adoption d'un acte par le Conseil par un vote majoritaire ${ }^{73}$. Le recours à une base juridique cumulative se justifie dans la présente hypothèse à plus d'un titre. Tout d'abord, il s'agit de mettre en sommeil la stricte légalité communautaire ; en cas contraire, la procédure de co-décision est le plus souvent de règle pour la formation de la volonté du législateur communautaire et il convient de compenser ici la moindre participation du Parlement par une exigence procédurale supérieure du côté du Conseil. En outre, si le nouvel article 299 § 2 contient une abondante énumération des circonstances justifiant des dispositions différenciées, il ne prévoit pas expressément la suspension, même partielle et temporaire, de l'application du droit primaire commun ; on peut alors admettre qu' on se trouve dans la situation où, « l'objet visé par l'action communautaire (résulte) d'une disposition particulière du traité », mais

73. Sur cette tendance, v. Joël Rideau, Droit institutionnel de l'Union et des Communautés européennes, coll. Manuels, LGDJ, $2^{\mathrm{e}}$ éd. 1996, p. 102-103; Anne Wachsmann, op. cit., p. 2, et par ex., CJCE, 26 mars 1987, Commission c/ Conseil, aff. 45/86, Rec. p. 1517 ; CJCE, 30 mai 1989, Royaume-Uni c/ Conseil, aff. 56/88, Rec. p. 1615. Sur la nécessité d'une double base juridique incluant l'article 235, CJCE, 30 mai 1989, Commission c/ Conseil, aff. 242/87, points 33 à 37, Rec. p. 1425. 
où les «pouvoirs... octroyés à la Communauté... ne lui permettent pas de "couvrir" l'ensemble des dispositions figurant dans une mesure à adopter ${ }^{74}$. Cette catégorie de «mesures spécifiques » évoquées par l'article 299 § 2 peut trouver sa base juridique dans le nouvel article 308 (ex-article 235), alors que la référence à l'article 299 § 2 se justifie pour expliquer la nécessité de remédier aux contraintes particulières des régions en cause ou de les compenser. Enfin, à propos de la validité de l'autorisation donnée par le Conseil de pratiquer des exonérations d'octroi de mer respectant des conditions strictes, la Cour conclut que l'examen de la décision 89/688 - simultanément fondée sur les articles $227 \S 2$ et 235 alors en vigueur - «n'a fait apparaître aucun élément de nature à affecter sa validité ${ }^{75}$ et elle s'abstient cette fois de considérer l'article 235 comme une base juridique inappropriée ${ }^{76}$.

La présentation qui vient d'être proposée d'une lecture du nouvel article $299 \S 2$, à la lumière de pistes tracées par une jurisprudence rendue avant l'entrée en vigueur du traité de révision, se heurte à l'argument du changement de contenu du droit primaire. La présente étude cherche à dépasser l'énoncé d'incertitudes - en attendant que la Cour statue - pour déceler les indices d'une possible conciliation entre la reconnaissance de la légitimité de la différenciation et les exigences de l'appartenance à un ordre juridique orienté vers l'intégration, en cohérence avec une ligne jurisprudentielle antérieure $^{77}$. Ce résultat, sans doute décevant pour ceux qui ont cherché à faire modifier le droit primaire dans un sens plus favorable à la différenciation, souligne que la rédaction passablement confuse de

74. Catherine Flaesch-Mougin, «Article 235 », in Vlad Constantinesco et autres (dir.), Traité instituant la CEE - Commentaire article par article, Economica, 1992, p. 1509 et s. (spécialement, p. 1512, p. 1515).

75. Arrêt Chevassus-Marche précité, point 54 et dispositif.

76. En sens inverse, sur l'impossibilité de fonder valablement sur l'article 235 l'article 4 de la décision 89/688 précitée, voir CJCE, 9 août 1994, Lancry, aff. C-363/93, point 37, Rec. p. I-3957 ; sur un plan plus général, CJCE, avis 2/94 du 28 mars 1996, point 35, Rec. p. I-1759.

77. Ce ne serait pas la première fois qu'une juridiction suprême réduirait autant qu'il est possible la portée de mutations constitutionnelles ; voir Georges Vedel, Pierre Delvolvé, Droit Administratif, tome 1, PUF, Thémis, 1990, p. 38. 
l'article 299 § 2 mérite d'être reprise, soit pour préciser expressément que le statut de pleine intégration juridique tolère des altérations ponctuelles s'apparentant à la catégorie des mesures de sauvegarde, soit pour instituer un statut ouvertement dérogatoire, à l'instar de ceux qui figurent aux paragraphes 5 et 6 du même article. Cette dernière éventualité n'a jamais figuré parmi les revendications des porte-parole des régions concernées ${ }^{78}$, mais l'expérience de la Conférence intergouvernementale de 1996 peut mieux faire comprendre à quel point il est difficile de faire admettre par l'unanimité des États membres le cumul des avantages de l'intégration et de son contraire. $\mathrm{Au}$ moment où la perspective d'une autre révision se profilait avant l'adhésion de nouveaux États candidats ${ }^{79}$, il aurait pu être utile de faire inscrire la clarification souhaitée à l'ordre du jour.

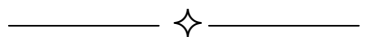

\section{Résumé}

La refonte du droit communautaire primaire relatif au statut de sept régions ultramarines de la Communauté européenne a été réclamée dès 1995, par les Présidents des Exécutifs régionaux concernés, en vue de desserrer les contraintes juridiques précédentes, mais dans le souci de préserver les avantages tirés $\mathrm{du}$ droit commun. La rédaction du nouvel article 299 § 2 du traité $\mathrm{CE}$, à
Abstract

The reshaping of "primary" EC law concerning the seven overseas European Community regions was claimed from 1995 by their executives in order to get easier "adaptations" to the common Community law. The new article 299 \& 2 of the EC Treaty appears to be disappointing as it doesn't solve uncertainties about the degree of legal differentiation now allowed for the "outermost regions".

78. Outre les prises de position précédant la rédaction définitive du traité d'Amsterdam citées supra, voir aussi : Déclaration finale de la Réunion des sept Présidents des Régions ultrapériphériques, Remire-Montjoly (Guyane), 5 mars 1999.

79. Voir « Protocole sur les institutions dans la perspective de l'élargissement de l'Union européenne » (Protocole $\mathrm{n}^{\circ} 11$ annexé au traité sur l'Union européenne et aux traités communautaires par le traité d'Amsterdam), art. 2, (Union européenne, Traité d'Amsterdam, Luxembourg, OPOCE, 1997, p. 111). 
la suite de l'entrée en vigueur du traité d'Amsterdam le $1^{\text {er }}$ mai 1999, s'avère décevante. En effet, par-delà une rédaction pour le moins confuse, elle laisse planer bien des incertitudes sur le degré de différenciation désormais licite à l'intérieur d'un ordre juridique tourné vers l'intégration.

La présente étude s'attache à mettre en lumière que si ces régions peuvent bénéficier au moins des mêmes possibilités de différenciation juridique que la plupart des régions européennes - au prix d'exigences de procédure souvent complexes -, on peut parler d'un statut propre aux sept régions ultrapériphériques dans la mesure où sont tolérés des écarts supplémen-taires par rapport au droit commun.

Cependant, si la légitimité de dispositions particulières est amplement explicitée dans la nouvelle rédaction, celle-ci évoque des limites qu'il serait illicite de transgresser, par le rappel de l'obligation de ne pas nuire à « l'intégrité et à la cohérence de l'ordre juridique communautaire ». La jurisprudence récente de la Cour de Justice peut fournir des pistes sur la possible conciliation entre le principe d'intégration et des altérations tolérables à la portée de clauses du traité. Le régime juridique n'étant pas celui de la dérogation et les exigences de procédure pouvant s'avérer plus complexes que ce qui découle de la première lecture de la nouvelle stipulation, une clarification semble souhaitable à l'occasion d'une prochaine révision.
The paper first shows that these regions are at least eligible to the same degree of legal differentiation likely to be set up for common European regions. It is then looking on eventual special differentiation, and shows that although art. $299 \quad \$ 2$ is clearly asserting legitimacy of special measures, it nevertheless strongly reaffirms the obligation not to undermine "integrity and coherence of the Community legal order". Actually, the new legal regime is not a "derogative" one but it allows safeguard measures, and procedure to use may turn out to be heavier than expected. 
Mots-clés

Açores - Canaries - Guadeloupe -

Guyane - Madère - Martinique - Réunion - Traité d'Amsterdam - Ordre juridique communautaire - Principe d'intégration Différenciation - Modulation - Dérogation - Mesure de sauvegarde - Base juridique.
Keys-words

Azores - Canaries - Guadeloupe - French Guyana-Madeira - MartiniqueReunion-Amsterdam Treaty-Community legal order - Integration Principle -

Differentiation -Modulation - Derogation - Safeguard Measures - Legal Ground 